Revue d'histoire de l'Amérique française

REVUE D.HISTOIRE DE L'AMÉRIQUE FRANÇAISE

\title{
L’électrification du monde rural québécois
}

\section{Marie-Josée Dorion}

Volume 54, numéro 1, été 2000

URI : https://id.erudit.org/iderudit/305653ar

DOI : https://doi.org/10.7202/305653ar

Aller au sommaire du numéro

\section{Éditeur(s)}

Institut d'histoire de l'Amérique française

\section{ISSN}

0035-2357 (imprimé)

1492-1383 (numérique)

Découvrir la revue

\section{Citer cet article}

Dorion, M.-J. (2000). L'électrification du monde rural québécois. Revue d'histoire de l'Amérique française, 54(1), 3-37. https://doi.org/10.7202/305653ar

\section{Résumé de l'article}

À la fin des années 1920, une faible minorité d'exploitations agricoles québécoises dispose du service de l'électricité, et les distributeurs privés ne prolongent que très lentement leurs réseaux en milieu rural. À la faveur d'un vaste mouvement d'opposition aux « trusts ", l'électrification rurale devient bientôt un véritable enjeu politique et social. Jusqu'en 1945 cependant, l'électrification des fermes québécoises progresse peu, et l'écart avec l'Ontario ne cesse de s'accroître. Cet article met en perspective les revendications d'intellectuels et de syndicalistes ainsi que les diverses mesures législatives adoptées par l'État entre les années 1920 et le début des années 1960. Il appert qu'après 1945, l'Office de l'électrification rurale agit comme catalyseur en matière d'électrification rurale. Mais ce sont bien autant les changements socio-économiques de l'après-guerre qui ont permis l'électrification accélérée du monde rural. Entre 1945 et 1955, les efforts conjugués des entreprises et des nouvelles coopératives feront multiplier par trois la proportion d'exploitations électrifiées, malgré la persistance de profondes inégalités régionales. L'étude détaillée de la diffusion de l'électricité dans les comtés fortement ruraux de Nicolet et de Yamaska confirme, entre autres, l'importance de la densité de l'habitat dans l'électrification des zones rurales. 


\title{
L'électrification
}

\section{du monde rural québécois ${ }^{1}$}

\author{
MARIE-JOSÉE DORION \\ Centre d'études québécoises \\ Université du Québec à Trois-Rivières
}

RÉsumÉ - À la fin des années 1920, une faible minorité d'exploitations agricoles québécoises dispose du service de l'électricité, et les distributeurs privés ne prolongent que très lentement leurs réseaux en milieu rural. À la faveur d'un vaste mouvement d'opposition aux «trusts», l'électrification rurale devient bientôt un véritable enjeu politique et social. Jusqu'en 1945 cependant, l'électrification des fermes québécoises progresse peu, et l'écart avec l'Ontario ne cesse de s'accroître. Cet article met en perspective les revendications d'intellectuels et de syndicalistes ainsi que les diverses mesures législatives adoptées par l'État entre les années 1920 et le début des années 1960. II appert qu'après 1945, l'Office de l'électrification rurale agit comme catalyseur en matière d'électrification rurale. Mais ce sont bien autant les changements socio-économiques de l'après-guerre qui ont permis l'électrification accélérée du monde rural. Entre 1945 et 1955 , les efforts conjugués des entreprises et des nouvelles coopératives feront multiplier par trois la proportion d'exploitations électrifiées, malgré la persistance de profondes inégalités régionales. L'étude détaillée de la diffusion de l'électricité dans les comtés fortement ruraux de Nicolet et de Yamaska confirme, entre autres, l'importance de la densité de l'habitat dans l'électrification des zones rurales.

1. Cet article est inspiré de notre mémoire de maitrise, L'électrification rurale $d u$ Centre $d u$ Québec, rive sud du fleuve, 1920-1963, Université du Québec à Trois-Rivières, 1997. Nos remerciements vont à Claude Bellavance, Normand Séguin et Sonia Dorion pour leurs commentaires sur les versions préliminaires de cet article ainsi qu'au FCAR et au CRSHC. 
ABSTRACT - By the end of the 1920's, only a small fraction of Quebec farms had access to electrical services, and private suppliers extended their rural networks only very slowly. Rural electrification soon became a locus of political and social struggle through widespread opposition to «trusts». By 1945, the gap in rural electrification between Quebec and Ontario had continued to widen. This article analyses the claims of intellectuals and syndicalists concerned by rural electrification, as well as the numerous legislative measures adopted by the State between 1920 and the early 1960s. After 1945, the Rural Electification Board appears to have acted as a catalyst, but post-war social and economic changes also contributed to the process of rural electrification. Between 1945 and 1955 , the joint efforts of firms and new cooperatives helped triple the proportion of farms having access to electricity, though without eliminating deep regional inequalities attributable in part to differences in population density, as suggested by detailed studies of the rural counties of Nicolet and Yamaska.

\section{INTRODUCTION}

L'électrification du monde rural est demeurée longtemps un aspect mal connu de l'histoire de l'électricité. Depuis quelques années toutefois, cette thématique jouit d'un renouveau d'intérêt dans les pays occidentaux, où elle a déjà suscité d'abondantes recherches ${ }^{2}$. Au Canada ${ }^{3}$, c'est le cas ontarien qui a été le mieux étudié grâce à la parution de

2. Pour la France, on consultera, notamment, Jean-Claude Felder, "L'électrification rurale et son financement de 1946 à nos jours", dans Fabienne Cardot, dir., Le financement de l'industrie électrique, 1880-1980 (Paris, AHEF/PUF, 1991), 115-129, Actes du colloque de l'Association pour l'histoire de l'électricité en France; Martine Muller, "L'électrification rurale: Frolois, 19191939», Bulletin d'histoire de l'électricité, 6 (décembre 1985): 61-74; Thierry Nadau, "Le financement de l'électrification rurale dans l'entre-deux-guerres", dans Fabienne Cardot, dir., op. cit. En Grande-Bretagne, Leslie Hannah, Electricity Before Nationalization: A Study of the Development of the Electricity Supply Industry in Britain Before 1948 (London, Macmillan Press, 1979), 189-192. Pour les États-Unis, se référer, entre autres, à Deward C. Brown, Electricity for Rural America. The Fight for the REA (Westport, Greenwood Press, 1980), 178 p. ; FrankJ. Busch, History of Montana Rural Electric Cooperatives, 1936-1971, thèse de doctorat, University of Montana, 1975 ; David E. Nye, Electrifying America : Social Meanings of a New Technology (Cambridge, The MIT Press, 1991), chapitre 7, "Rural Lines»; et James C. Williams, "Otherwise a Mere Clod: California Rural Electrification ", IEEE Technology and Society Magazine, 7,4 (décembre 1988): 13-29.

3. Pour l'ensemble du Canada, voir, notamment, Melvin Baker, "Rural Electrification in Newfoundland in the 1950s and the Origins of the Newfoundland Power Commission", Newfoundland Studies, 6,2 (1990): 190-209; David A. Schulze, The Politics of Power: Rural Electrification in Alberta, 1920-1989, mémoire de maîtrise, Université McGill, 1989, 188 p. ; L. C. Volk, The Social Effects of Rural Electrification in Saskatchewan, mémoire de maîtrise, Université de Regina, 1980, 220 p. On peut aussi consulter Christopher Armstrong et Henry Vivian Nelles, Monopoly's Moment: The Organization and Regulation of Canadian Utilities, 1830-1930 (Toronto, University of Toronto Press, 1986), 393 p., bien que ce volume ne traite pas spécifiquement d'électrification rurale. 
Power at $\mathrm{Cost}^{4}$, un ouvrage qui analyse le rôle central joué par HydroOntario dans l'électrification des campagnes. K. R. Fleming y aborde plusieurs questions d'importance, comme les défis que représentent l'intégration du monde rural au marché de l'électricité et le rapport entre le prix de l'électricité et le développement de la consommation des ménages. On ne dispose pas de travaux semblables pour le Québec. L'électrification rurale n'y a pas encore attiré beaucoup l'attention des historiens. Ainsi, dans le premier ouvrage consacré à l'histoire de l'électricité au Québec, Hydroelectricity and Industrial Development in Quebec, 1898-19405, publié par J. H. Dales en 1957, le thème de l'électrification rurale est presque absent. Claude Bellavance, dont les récents travaux ${ }^{6}$ jettent les bases d'une meilleure connaissance du processus d'électrification, ne fait par ailleurs lui-même qu'effleurer la problématique de l'électrification rurale. En fait, Yves Tremblay est le seul historien à s'être véritablement penché sur le sujet. Sa thèse ${ }^{7}$ retrace l'électrification d'une région fortement rurale : le Bas-Saint-Laurent. Elle montre notamment que cette région a été particulièrement marquée par les activités de l'Office de l'électrification rurale, créé en 1945. Dans un article ultérieur, Tremblay propose un survol des politiques du gouvernement québécois en matière d'électrification rurale entre 1935 et $1964^{8}$. Si ces travaux ont posé les premiers jalons d'une histoire de l'électrification rurale au Québec, bien des aspects de ce processus complexe demeurent méconnus, à commencer par les bases matérielles sur lesquelles il s'appuie et le contexte sociopolitique dans lequel il s'inscrit. Tremblay, tout en reconnaissant le compromis que représente la mise

4. Keith R. Fleming, Power at Cost, Ontario Hydro and Rural Electrification, 1911-1958 (Montréal, McGill-Queen's University Press, 1992), 326 p.

5. J. H. Dales, Hydroelectricity and Industrial Development in Quebec, 1898-1940 (Cambridge, Harvard University Press, 1957), 269 p.

6. Voir, en particulier, Claude Bellavance, Shawinigan Water \& Power, 1898-1963. Formation et déclin d'un groupe industriel au Québec (Montréal, Boréal, 1994), 448 p. ; "Réseaux, territoires et électricité : la dynamique spatiale du processus d'électrification du Québec méridional», dans Serge Courville et Normand Séguin, dir., Espace et culture (Sainte-Foy, Les Presses de l'Université Laval, 1995), 393-404; “L'État, la houille blanche et le grand capital. L'aliénation des ressources hydrauliques du domaine public québécois au début du $\mathrm{xx}^{\mathrm{e}}$ siècle ", Revue d'histoire de l'Amérique française, 51,4 (printemps 1998): 487-520.

7. Yves Tremblay, Histoire sociale et technique de l'électrification au Bas-Saint-Laurent, 1888-1963, thèse de doctorat, Université Laval, 1992, 581 p. et "Le monde rural et l'électricité ", Revue d'histoire du Bas-Saint-Laurent, 17,1 (janvier 1994) : 25-29.

8. Yves Tremblay, «Entre le privé et le public : l'électrification rurale au Québec, 1935-1964 », Bulletin d'histoire de l'électricité, 22 (décembre 1993): 173-185. 
sur pied de l'OER, fait par ailleurs une part belle au gouvernement Duplessis, ce qu'une analyse rigoureuse des faits permet difficilement de soutenir. Comme nous le verrons dans la partie B de cet article, c'est l'ensemble d'un contexte nouveau qui se met en place dans l'aprèsguerre qui a permis l'électrification des exploitations agricoles et non pas seulement la création de l'Office par le gouvernement Duplessis.

La recherche dont nous livrons ici les résultats appréhende l'électrification rurale à deux niveaux. Empruntant la voie de l'analyse sociopolitique, elle tente d'abord de rendre compte des débats dont la question de l'électricité en milieu rural a fait l'objet. Une attention particulière est accordée à l'entre-deux-guerres ${ }^{9}$, une période marquée par la montée du sentiment antimonopolistique et par la recherche de nouveaux modèles, que d'aucuns n'ont pas hésité à qualifier de première révolution tranquille. Entre 1930 et 1945, en effet, l'électricité, et l'électrification rurale sont au cœur d'un vaste débat auquel prend part une fraction de plus en plus large de la population, qui considère désormais l'électricité à la fois comme un outil collectif de développement économique et comme un service public à part entière.

Dans cette perspective, plusieurs grandes questions ont orienté notre étude. Dans quel contexte l'électrification rurale du Québec est-elle devenue un enjeu politique ? À partir de quel moment les agriculteurs ont-ils considéré l'électricité comme un bien essentiel ? L'analyse systématique des rapports et des ordonnances des commissions de l'État ${ }^{10}$, de même que de la correspondance avec les usagers et les compagnies nous permet d'aborder de telles questions. Par ailleurs, la reconstitution du cadre législatif et réglementaire ${ }^{11}$ nous renseignera sur l'approche privilégiée par les divers gouvernements qui se sont succédé depuis les

9. Claude Bellavance, Roger Levasseur et Yvan Rousseau, "De la lutte antimonopoliste à la promotion de la grande entreprise; l'essor de deux institutions économiques au Québec: Hydro-Québec et Desjardins, 1920-1965", Recherches sociographiques, 2 (1999): 551-578; voir aussi, Fernand Dumont, Jean Hamelin et Jean-Paul Montminy, Les idéologies au Canada français : 1930-1939 (Québec, Les Presses de l’Université Laval, 1978), 361 p.

10. Les sources disponibles aux Archives d'Hydro-Québec sont variées. Nous y avons consulté, notamment, les rapports annuels de l'Office de l'électrification rurale (OER) et le fonds de la Régie de l'électricité, riche de quelques centaines de titres. Outre les rapports et les ordonnances de la Régie, on y trouve les nombreuses études exécutées sur l'électrification rurale ainsi que les échanges entre les entreprises d'électricité et la Régie sur le même sujet. Les rapports annuels des distributeurs privés, ceux de Shawinigan Water \& Power (SWP), de Southern Canada Power et de City Gas \& Electric Co. ont également été mis à contribution.

11. Le cadre législatif de l'électrification rurale a été restitué grâce au repérage et à l'analyse de l'ensemble des lois pertinentes sanctionnées par la législature québécoise depuis la fin du XIx siècle jusqu'en 1964 . 
années 1920 jusque dans l'après-guerre: comment l'État encadrait-il les pratiques commerciales des distributeurs privés? Quel rôle le gouvernement Godbout entendait-il donner à Hydro-Québec en matière d'électrification rurale lors de la première nationalisation de 1944 ? Dans quel contexte l'État québécois a-t-il été amené à soutenir la création de coopératives d'électricité ? Comment ont évolué les politiques gouvernementales en matière d'aide financière à l'érection de lignes rurales ?

Notre approche nous conduit également à déborder la seule dimension sociopolitique pour mettre en lumière certaines modalités techniques et économiques de l'électrification des campagnes. L'électrification rurale nécessite le déploiement dans l'espace d'importantes infrastructures de transport et de distribution, lesquelles sont reliées à des équipements de production d'énergie de plus en plus centralisés à partir des années $1920^{12}$. À un premier niveau, l'analyse à l'échelle régionale et suprarégionale servira d'abord à saisir globalement l'avancée de l'électricité vers la campagne entre 1920 et le début des années 1960. Il s’agira notamment de préciser l'ampleur du retard du Québec par rapport à l'Ontario, puis de mesurer et d'interpréter, à l'échelle des comtés cette fois, les rythmes différenciés de la diffusion de l'électricité en milieu rural. Nous examinerons aussi le processus d'électrification à l'échelle infrarégionale. À cette fin, les comtés de Nicolet et de Yamaska - un territoire ayant conservé un caractère rural accusé jusqu'aux années 1960 - ont été choisis. Nous chercherons à déterminer dans quelle mesure le développement des marchés urbains et villageois a été un préalable à l'électrification des rangs. Nous tenterons aussi de vérifier si le contexte sociopolitique a pu inciter les distributeurs privés à modifier leur politique commerciale et, partant, à accélérer leur programme d'extension de réseau à la campagne. Pour aborder ces questions, nous nous appuyons principalement sur une source encore inexploitée, les "rapports d'électrification rurale ${ }^{13}$ " soumis annuellement à la Régie de l'électricité par les distributeurs privés.

12. Claude Bellavance, Shawinigan Water \& Power..., op. cit., 75-134.

13. Ces rapports recèlent plusieurs types d'informations: 1 - pour chaque année concernée, un résumé du programme d'électrification rurale de la compagnie avec les lignes à construire par division de recensement, localité et même par rang ; 2 - le rapport de construction, c'est-àdire le nombre de milles de lignes rurales ajouté au réseau de l'entreprise; 3 - et la correspondance entre les dirigeants de la Régie et ceux de la compagnie. Ces documents nous renseignent non seulement sur les progrès réalisés dans chacune des paroisses de Nicolet et de 
Nous n'avons pas cherché cependant à mesurer l'impact de l'électrification des fermes sur la modernisation de l'agriculture. Il s'agit là d'un problème complexe qui soulève maintes questions méthodologiques, lesquelles sont au centre de nos recherches en cours.

Cet article se développe en deux parties, chacune correspondant à des phases contrastées de l'histoire de l'électrification rurale du Québec. La première, qui s'étend des années 1920 (érection des premiers réseaux ruraux) à la fin du Second Conflit mondial, est caractérisée par la lenteur de la diffusion, de même que par l'intensification du "problème" de l'électrification rurale. La seconde partie est consacrée aux années 1945 à 1963, alors que les conditions semblent désormais réunies pour l'électrification rapide des fermes dans la plupart des régions du Québec, malgré la politique toujours peu favorable à l'intervention directe de l’État mise de l'avant par le gouvernement Duplessis.

\section{UN LENT DÉMARRAGE SOUS LE RÉGIME DES ENTREPRISES PRIVÉES}

À la fin des années 1920, alors que l'électricité fait partie du quotidien de la plupart des foyers urbains depuis presque une génération, rares sont les exploitations agricoles qui ont accès à ce service ${ }^{14}$. Le peu d'attention qu'accorde alors le gouvernement québécois à l'électrification tranche avec la situation qui prévaut en Ontario. Les mesures avant-gardistes adoptées par l'État ontarien en matière d'électrification rurale étonnent et suscitent l'envie.

\section{Comparaison entre le Québec et l'Ontario}

Durant les trois premières décennies du siècle, en effet, l'Ontario fait figure de pionnière à l'échelle nord-américaine. Hydro-Ontario, entreprise d'État fondée en 1906, détient le monopole du transport d'électricité et va graduellement devenir le principal producteur d'énergie, tandis que les municipalités assurent la vente au détail. Et alors qu'il est pris à partie par le lobby des entreprises privées nord-américaines ${ }^{15}$, le

Yamaska entre 1936 et 1962, mais ils permettent aussi de mieux comprendre l'évolution des relations entre la Régie et SWP. Grâce à ce dossier, nous sommes, entre autres, en mesure d'évaluer les efforts consentis par l'entreprise pour l'électrification des campagnes tout au long de la période.

14. À peine 1 sur 24 en 1921 selon le recensement décennal du Canada (RDC), 1921.

15. La National Electric Light Association (NELA) est une organisation des compagnies privées de gaz et d'électricité américaines. Son but est de combattre la régie publique des services électriques. Hydro-Ontario lui sert souvent de cible. Philippe Hamel, Le trust de l'électricité, agent de corruption et de domination, centre de la dictature économique. Mémoire présenté 
régime public ontarien sert de référence pour les mouvements réformistes qui réclament la création d'un système semblable ${ }^{16}$.

L'État ontarien s'intéresse à l'électrification rurale dès 1911. Ce n'est toutefois qu'à partir de 1920-1921, par le biais d'une série de mesures législatives, qu'Hydro-Ontario inaugure un programme qui bénéficie du soutien financier du gouvernement ontarien. La plus importante de ces lois est la Rural Hydro-Electric Distribution Act (1921) par laquelle l'État s'engage à assumer jusqu'à $50 \%$ du coût global du réseau rural, de l'extrémité de la ligne existante jusque chez le consommateur. Ces mesures stimulent grandement l'électrification des campagnes ontariennes, particulièrement pendant les années 1930, alors que sévit la grande crise économique.

Le cas québécois contraste fortement avec le dynamisme ontarien. L'État n'offre aucun programme de subvention pour la construction de lignes rurales. Ce sont les distributeurs privés eux-mêmes, ou les services municipaux ${ }^{17}$, qui doivent assumer la totalité des coûts de construction des réseaux ruraux de transport et de distribution d'électricité. La comparaison entre le nombre d'exploitations agricoles électrifiées au Québec et en Ontario entre 1930 et 1942 (figure 1) ${ }^{18}$ illustre bien l'impact

à la Commission de l'électricité de la Province de Québec, 1934, 26. Il y a une section canadienne (Canadian Electrical Association), dont le représentant, en 1928, est J. B. Woodyatt de Southern Canada Power.

16. Une étude de 1929 sur l'électrification des fermes new-yorkaises établit une comparaison entre l'électrification rurale au Québec et en Ontario. La partie sur le Québec est peu développée par rapport à celle sur l'Ontario, laissant deviner que l'auteur a comparé les deux modèles et a retenu le plus intéressant. Roland F. Bucknam, An Economic Study of Farm Electrification in New-York, with a Discussion of Rural Electrification in the Provinces of Quebec and Ontario, Canada (Ithaca, Cornell University Agricultural Experiment Station, 1929), 65 p. David Nye souligne, quant à lui, que l'expérience d'Hydro-Ontario inspira, au cours des années 1920, le gouverneur de la Pennsylvanie qui souhaitait instaurer un système semblable. Mais il fut bloqué par les membres de la NELA et par des manœuvres politiques. David E. Nye, op. cit., 299. Dans les années 1930, tout comme le Québec, c'est en Ontario que l'Alberta va chercher les solutions possibles à l'électrification de ses exploitations agricoles. David Schulze, op. cit., 45. En 1945, la Saskatchewan engage un ingénieur d'Hydro-Ontario pour rédiger un rapport sur l'électrification rurale dans la province. L. C. Volk, op. cit., 43-77.

17. En 1943, la Régie des services publics estime que $98,1 \%$ de la consommation domestique d'électricité au Québec dépend alors des distributeurs privés. Le reste est assuré par les services municipaux. Dans Rapports et statistiques: recensements des clientèles potentielles, Archives d'HydroQuébec (AHQ), fonds de la Régie provinciale de l'électricité (fonds P3), document $n^{\circ}$ P3/219.

18. La Régie est d'avis que les données des recensements correspondent à celles des autres sources de renseignements dans les régions où l'électrification est assez avancée, mais qu'ils tendent à surestimer la réalité dans les régions peu électrifiées. C'est pourquoi nous utilisons les chiffres (corrigés) fournis par la Régie. Dans Rapports sur l'électrification rurale dans les autres provinces canadiennes, $\mathrm{AHQ}$, fonds $\mathrm{P} 3$, document $\mathrm{n}^{\circ} \mathrm{P} 3 / 232$. 
FIGURE

\section{Les exploitations agricoles électrifiées au Québec et en Ontario, 1930-1942}

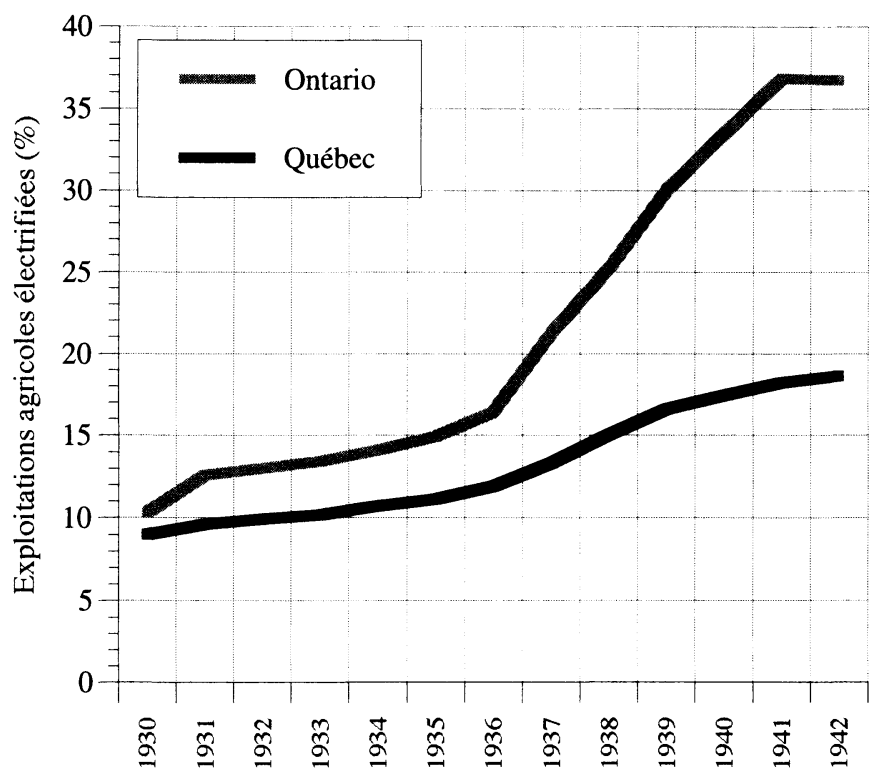

Source : AHQ, P3/217

favorable des dispositions ontariennes. En $1931^{19}$, le pourcentage de fermes électrifiées au Québec (9,6\%) est voisin de celui de l'Ontario $(12,6 \%)$. Mais la situation est tout autre en $1941^{20}$. À peine $18 \%$ des

19. "On a convenu un peu partout de prendre comme indice d'électrification rurale le pourcentage des fermes ayant le service d'électricité. Cet indice est sujet aux restrictions suivantes: a) le nombre de logements ne correspond pas toujours au nombre de fermes; b) le long des lignes rurales, il y a d'autres clients que les cultivateurs. De plus, on ne s'entend pas toujours sur la définition d'une ferme. [Hydro-Ontario considère comme ferme les exploitations agricoles de cinq acres et plus, alors que le recensement compte des fermes d'un ou deux acres.] Néanmoins, sans être parfait, l'indice a sa valeur." Dans Statistiques générales, analyses et comparaison avec l'électrification rurale de l'Ontario, AHQ, fonds $\mathrm{P} 3$, document $\mathrm{n}^{\circ} \mathrm{P} 3 / 217$.

20. L'électrification rurale dans les autres provinces canadiennes, selon le RDC de 1941 produit les pourcentages suivants: Île-du-Prince-Édouard, 5,4\%; Nouvelle-Écosse, $26 \%$; Nouveau-Brunswick, 18,5\%; Manitoba, 7,3\%; Saskatchewan, 4,7\%; Alberta, 5,4\% et Colombie-Britannique, $35,8 \%$; pour une moyenne de $19,8 \%$ pour l'ensemble du Canada. Le Québec, comparé à la moyenne canadienne, semble en assez bonne position. Plusieurs éléments amoindrissent cependant considérablement cette apparente bonne position. Notons d'abord que la situation des Prairies est très différente des autres, en raison de la nouveauté de son peuplement et surtout de la répartition des fermes sur le territoire. Comparé à la Colombie- 
exploitations agricoles québécoises disposent alors du service, tandis qu'en Ontario, $36,8 \%$ sont desservies.

Jusqu'en 1935, en Ontario comme au Québec, le rythme d'électrification est plutôt lent. Par la suite, l'écart entre les deux provinces s'accentue. De 1935 à 1942, l'Ontario enregistre un gain annuel moyen de 3,5\%, comparativement à $1,5 \%$ pour le Québec. À ce rythme, seule une faible minorité d'exploitations agricoles québécoises aura accès à l'électricité avant 1945.

\section{Les contrastes de l'électrification rurale dans le Québec de l'entre-deux-guerres}

La comparaison avec l'Ontario met en évidence le retard croissant du Québec en matière d'électrification rurale avant 1945. Cette première évaluation cache elle-même d'importantes disparités.

Les divisions de recensement, qui se superposent aux comtés municipaux, nous fournissent une base de comparaison convenable pour apprécier la diversité régionale en matière d'électrification rurale durant l'entre-deux-guerres. Comme le révèle la cartographie de l'électrification des exploitations agricoles en $1931^{21}$, l'électricité est très inégalement répartie sur le territoire québécois (carte 1$)^{22}$. Quelques comtés sont bien desservis, d'autres, beaucoup plus nombreux, le sont faiblement, tandis que certains ne le sont à peu près pas. Pensons au comté de Papineau, par exemple, où à peine $3 \%$ des fermes sont électrifiées.

En plus d'être répandus de manière irrégulière, les réseaux ruraux sont géographiquement concentrés; ils desservent beaucoup mieux les régions centrales que la périphérie. Ces écarts reflètent bien l'impor-

Britannique, à la Nouvelle-Écosse et à l'Ontario, le Québec parait nettement sous-électrifié. Il ne faut également pas oublier que l'industrie électrique dans les Prairies et les Maritimes est très différente de celle de l'Ontario et du Québec. En comparaison, ces deux dernières provinces sont avantagées par une production d'électricité à grande échelle et par des réseaux de transport beaucoup plus développés. Ajoutons que les Prairies et Terre-Neuve feront face, jusque dans les années 1960 , à des problèmes d'approvisionnement en électricité.

21. Nous utilisons les données du recensement décennal, mais il faut souligner qu'il cumule les fermes qui disposent de l'éclairage électrique ou de l'éclairage au gaz. Nous avons présumé, tout comme Y. Tremblay, qu'il n'y avait pas de gaz disponible (sauf dans des cas très exceptionnels) sur les exploitations agricoles québécoises.

22. Les cartes ont été établies à partir des données du recensement décennal. Selon la Régie, comme nous l'avons vu, ces chiffres surévaluent quelque peu l'électrification des régions faiblement électrifiées. Ils permettent néanmoins de tracer un portrait fidèle de l'électrification des fermes québécoises par comté. L’analyse porte sur l'année 1931, car les données par comtés ne sont pas disponibles pour la période antérieure. Les fonds de carte ont été aimablement fournis par le Centre d'études québécoises de l'Université du Québec à Trois-Rivières. 


\section{CARTE 1}

\section{Les exploitations agricoles électrifiées au Québec, par comtés, en 1931}

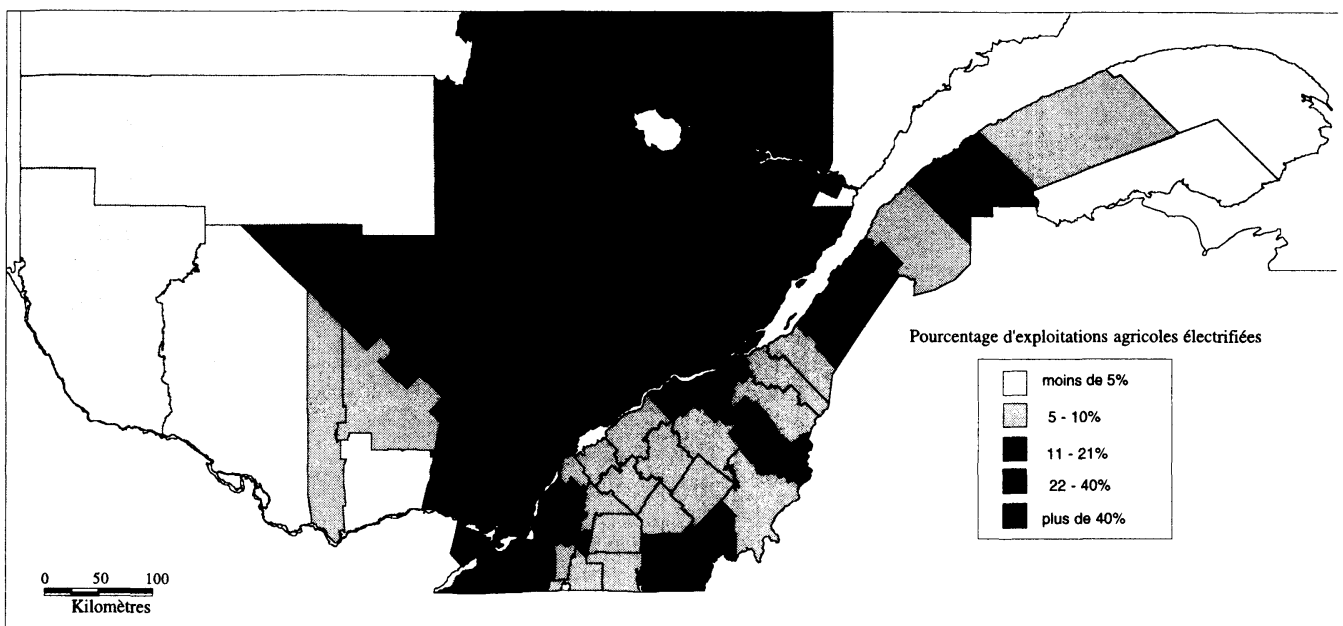

Source : Recensement du Canada, 1931.

Les classes des cartes ont été définies en fonction de la médiane pour chacune des années.

tance de la proximité des zones à plus grande densité de population, car on relie d'abord les lieux les plus populeux.

L'analyse de la carte suggère ainsi que, durant cette phase pionnière de l'électrification rurale, le proche voisinage des centres urbains est un facteur déterminant de la pénétration de l'électricité à la campagne. Les lignes rurales sont en fait des extensions des réseaux urbains que les distributeurs privés prolongent en dehors de l'aire citadine pour rejoindre des marchés prometteurs. Les comtés qui entourent Montréal en constituent des exemples convaincants. Ils présentent le plus haut pourcentage d'exploitations électrifiées de la province: Île de Montréal/ Île Jésus ( $73 \%$ ), L'Assomption ( $44 \%$ ) et Chambly (35\%). En dehors de l'aire montréalaise, la présence des centres régionaux a la même influence positive sur l'électrification des fermes. Les comtés de Québec (40\%), de Champlain (29\%) et de Chicoutimi (26\%) affichent tous des taux d'électrification de beaucoup supérieurs à la moyenne québécoise ${ }^{23}$.

23. Quatorze pour cent selon le RDC de 1931 et $10 \%$ selon la Régie, dans op. cit., document $\mathrm{n}^{\circ} \mathrm{P} 3 / 217$. 
Ce constat nous amène à formuler une hypothèse concernant le retard du Québec par rapport à l'Ontario. Certes, l'avance ontarienne est en partie imputable à des interventions étatiques. Mais ne pourrait-elle pas être aussi reliée à la densité plus grande du réseau urbain ontarien ${ }^{24}$ ?

Si tel était le cas, le tissu urbain plus lâche du territoire situé au sud du fleuve Saint-Laurent pourrait expliquer son net désavantage par rapport à la rive nord, exception faite des comtés du sud de Montréal. L'électrification des Cantons-de-l'Est, notamment, est plutôt lente et ce, malgré plusieurs facteurs positifs. En effet, la construction précoce d'un réseau électrique dans cette région, la présence d'une compagnie d'envergure régionale (Southern Canada Power), de même que l'existence d'un marché industriel prospère, ne semblent pas avoir profité à l'électrification des exploitations agricoles. La région dispose d'un réseau hydrographique relativement pauvre et les installations de production demeurent de faible capacité, alors qu'au nord, Shawinigan Water $\&$ Power (SWP) produit de grandes quantités d'énergie depuis le début du siècle. L'immense potentiel hydro-électrique de la région mauricienne a permis, dès les années 1900, des économies d'échelle importantes. L'énergie abondante a poussé la compagnie à envisager très tôt l'élargissement de son marché à l'extérieur de la Mauricie. Attirée par le plus gros marché du Québec, SWP a construit dès les années 1903-1904 une ligne à haute tension vers Montréal. Cette ligne rendait l'électricité accessible aux municipalités situées sur son parcours. Bien entendu, il fallait d'abord abaisser le voltage et ériger quelques lignes de distribution. Plusieurs localités de moyenne et de petite tailles ont ainsi été desservies entre 1910 et 1930 . Tous ces éléments montrent l'importance des centres urbains et industriels dans la diffusion de l'électricité à la campagne.

24. Pierre Lanthier a d'ailleurs déjà noté la différence entre les patterns d'urbanisation pour expliquer les choix du Québec et de l'Ontario en matière de propriété des ressources hydroélectriques: "L'industrie électrique entre l'entreprise privée et le secteur public, le cas de deux provinces canadiennes: 1890-1930», F. Cardot, dir., Un siècle d'électricité dans le monde (Paris, Presses universitaires de France, 1987), 23-36. Mentionnons également que le statisticien de SWP (Huet Massue) soutenait que les nombreux petits centres industriels en Ontario étaient responsables d'une meilleure diffusion de l'électricité à la campagne. Études des conditions affectant la consommation d'énergie électrique dans les provinces d'Ontario et de Québec (Montréal, The Shawinigan Water and Power Co., [s.d.], [1931]). 


\section{Les enjeux politiques de l'électrification rurale}

D'abord peu présent dans les débats publics, le retard de l'électrification rurale du Québec apparaît, à compter de 1930, de plus en plus inacceptable aux habitants des campagnes québécoises, au fur et à mesure qu'ils prennent conscience de l'importance de l'électricité pour la modernisation de l'agriculture ${ }^{25}$.

\section{Une nouvelle question d'actualité}

L'électrification rurale est une question complexe, car elle soulève à la fois des considérations morales (dans une société démocratique moderne, l'électricité est considérée comme un service public) et des considérations économiques. Durant l'entre-deux-guerres, l'électricité (production, transport et distribution) relève du secteur privé et est encore entièrement soumise aux mécanismes du marché. La recherche de profit conditionne donc la construction de toute nouvelle ligne. Les protestataires soutiennent que les compagnies d'électricité ont monopolisé des richesses naturelles appartenant à l'ensemble de la population et que, à ce titre, elles doivent aussi en faire bénéficier tous les citoyens. Ils jugent, en outre, que l'absence d'électricité à la campagne pénalise très lourdement le secteur agricole. La Crise qui débute en 1929 favorise cette prise de conscience. Elle entraîne une levée de boucliers à travers le Canada contre les grands "trusts» (du papier, du charbon et de l'électricité, notamment) que l'on tient responsables des difficultés de l'économie de marché et de la lenteur de la modernisation de l'agriculture.

La première manifestation publique en faveur de l'électrification des campagnes provient du milieu de l'enseignement agricole. En 1928, Charles Gagné, professeur d'économie rurale à l'École d'agriculture de Sainte-Anne-de-la-Pocatière, publie un article dans un quotidien de

25. Pour les contemporains, la modernisation agricole est largement synonyme de mécanisation des fermes. Nous savons qu'il s'agit plutôt d'une adaptation éminemment complexe de l'agriculture à l'économie de marché. L'augmentation des superficies cultivées, la baisse du nombre d'exploitants (par suite de la concentration des terres), l'abandon de certaines cultures domestiques au profit d'autres orientées vers le marché et la mécanisation des exploitations sont des indicateurs de cette modernisation. L'articulation de l'agriculture au marché - et les changements qu'elle commande dans la sphère agricole - ne s'est pas effectuée sans heurts. Perceptible dès le milieu du $\mathrm{xIx}^{\mathrm{e}}$ siècle, elle sera freinée par les crises successives qui frapperont le monde agricole d'avant 1945. La modernisation de l'agriculture s'accélérera après la fin des hostilités en Europe. Voir, entre autres, Normand Séguin, "L'agriculture de la Mauricie et du Québec, 1850-1950", RHAF, 35,4 (mars 1982): 537-562 ; Jean-Pierre Kesteman, Histoire du syndicalisme agricole au Québec: UCC-UPA, 1924-1984 (Montréal, Boréal Express, 1984), 327 p. 
Québec, L'Action catholique, où il réclame l'électrification des fermes et dénonce les abus des compagnies privées et leur manque d'intérêt face à la classe agricole $e^{26}$. L'économiste Esdras Minville fait sienne cette position dans un article sur Hydro-Ontario, publié en $1931^{27}$, où il réclame l'électrification rurale comme moyen d'augmenter la productivité des fermes.

À la même époque, Philippe Hamel ${ }^{28}$ s'engage dans une âpre lutte contre les firmes d'électricité. L'homme, qui prendra bientôt la tête d'un puissant mouvement de contestation et de revendication, dénonce au premier chef les tarifs élevés imposés par les distributeurs. Le début de la décennie 1930 est décisif pour le mouvement de contestation des «trusts» de l'électricité qui parvient alors à élargir ses bases sociales en milieu urbain ${ }^{29}$.

Les demandes des milieux agricoles - dont l'Union catholique des cultivateurs (UCC) est l'un des chefs de file - rejoignent les positions antimonopolistes des milieux urbains sur la critique du régime privé d'électricité, de même que sur les tarifs et les profits jugés trop élevés. $S$ 'ajoute une revendication qui les vise plus particulièrement: le droit pour tous les ruraux de disposer du service électrique.

\section{Les hésitations de l'État}

L'État hésite encore à imposer des limites à l'action des entreprises privées dans le champ de la distribution d'électricité. Le seul organisme public de réglementation, la Commission des services publics du Québec

26. Charles Gagné, L'Action catholique, 30 juin 1928, 17. C. Gagné réclame l'électrification rurale du Québec, mais son article porte surtout sur le fonctionnement du système ontarien et la différence entre ce dernier et le régime en vigueur au Québec. C'est Albert Rioux qui souligne que cet article est le premier portant sur l'électrification rurale. Nos recherches ne nous ont d'ailleurs pas permis de repérer de textes antérieurs à celui-ci dans les journaux. A. Rioux, L'électrification rurale du Québec (Sherbrooke, Imprimerie Le Messager St-Michel, 1942), 80.

27. Esdras Minville, "L'Hydro-Electric Commission de l'Ontario», L'Actualité économique (décembre 1931). Articles rassemblés dans E. Minville, La vie économique (Montréal, Fides/Les Presses des hautes études commerciales, 1981), 4 : 173-191.

28. Philippe Hamel, médecin et dentiste, sera député de l'Alliance libérale nationale (19351936) et député de l'Union nationale (1936-1939) pour le comté de Québec-Centre. Richard Lapointe, La politique au service d'une conviction. Philippe Hamel: deux décennies d'action politique, mémoire de maîtrise, Université Laval, août 1987, 167 p. À propos du mouvement contre les monopoles et des revendications de la ville de Québec pour municipaliser son service électrique, voir Daniel Boutet, Le mouvement d'opposition au monopole de l'électricité à Québec dans l'entre-deuxguerres, mémoire de maîtrise, Université du Québec à Trois-Rivières, avril 1999, 113 p.

29. Daniel Boutet, op. cit. et Claude Bellavance, Roger Levasseur et Yvan Rousseau, loc. cit. 
(CSPQ), dispose de pouvoirs relativement étendus ${ }^{30}$. Mais, par crainte de heurter les grandes entreprises productrices, il ne se résout pas à encadrer les pratiques monopolistes des grands distributeurs privés ni à les inciter à desservir les zones à faible densité de population. L'insatisfaction générale envers l'approche préconisée par l'État en matière de réglementation de l'industrie s'accentue entre 1930 et 1934 . Les attaques de plus en plus virulentes de Philippe Hamel, conjuguées aux demandes répétées des milieux agricoles, incitent finalement l'État québécois à se préoccuper des critiques formulées contre le régime de l'électricité ${ }^{31}$.

En 1934, il institue une commission présidée par Ernest Lapointe ${ }^{32}$. La commission est chargée de faire enquête sur le problème général de l'électricité et sur la question particulière de l'électrification rurale ${ }^{33}$. Une

30. La CSPQ, organisme chargé de réguler l'industrie électrique et créé en 1909, est habilitée par un amendement à sa loi constitutive (1911) à contraindre un distributeur privé à prolonger ses lignes dans les parties non desservies des municipalités. Elle a autorité pour intervenir dans " toutes contestations s'élevant sur la plainte d'une municipalité, à l'effet qu'une utilité publique faisant affaire dans telle municipalité ne fait pas bénéficier de son service une partie quelconque de cette municipalité [...]». Après audition des parties et enquête, "la commission peut ordonner l'extension de ce service et fixer les conditions de cette extension, [...]", Québec (province) SDQ : Loi amendant les Statuts refondus, 1909 concernant la Commission des services publics (1 Geo V, 1911, chapitre 14). C'est nous qui soulignons. La CSPQ a cependant comme pratique de ne pas employer ce pouvoir lorsque la ligne à construire est présumée non rentable pour le distributeur, c'est-à-dire lorsque les revenus anticipés annuellement ne couvrent pas les coûts de construction et d'entretien. Signalons que les agriculteurs et les municipalités rurales semblent peu enclins à s'adresser à cette commission avant 1931-1932. En 1930, par exemple, plus de 400 requêtes de toutes sortes ont été étudiées par la CSPQ; une vingtaine d'entre elles sont des demandes d'extension de service en milieu rural. Québec (province), Rapport annuel de la Commission des services publics de Québec pour l'année finissant le 30 juin 1930.

31. En 1930, l'État québécois s'enquiert officiellement de la situation de l'électrification rurale. Il charge l'ingénieur en chef de la Commission des services publics, A. Larivière, de préparer un rapport sur l'électrification rurale. Son diagnostic confirme les propos des milieux agricoles : l'électrification des campagnes est à peine commencée et elle ne pourra se faire sans l'intervention de l'État. Mais le gouvernement ne prend encore aucune mesure particulière pour accélérer la construction de lignes rurales. Alexandre Larivière, "Rapport sur les taux de l'électricité dans les campagnes et sur un projet de coopératives rurales de moteursélectriques ", 23 décembre 1930, AHQ, fonds C. Lapointe, document $\mathrm{n}^{\circ} \mathrm{P} 1 / 23$.

32. Ministre de la Marine et des Pêcheries (1921-1924) et ministre de la Justice (1924-1930 et 1935-1941) dans le cabinet de Mackenzie King. Voir John MacFarlane, Ernest Lapointe and Quebec's Influence on Canadian Foreign Policy (Toronto, University of Toronto Press, 1999), $270 \mathrm{p}$.

33. La Commission est chargée d'étudier les principaux points de mécontentement de la population: «[...] les conditions de l'électricité dans la province de Québec, et spécialement les questions suivantes: 1) Nationalisation, 2) Municipalisation, 3) Effets de la municipalisation des grands centres sur les districts ruraux de la province, 4) Les taux actuels de l'électricité et la possibilité de les réduire et 5) en général, la diffusion de l'électrification dans les municipalités rurales." Québec (province) Rapport de la Commission de l'électricité de la province de Québec au Premier ministre de la province, 21 janvier 1935, AHQ, fonds C. Lapointe, document $n^{\circ} \mathrm{P} 1 / 127,1$. 
vaste consultation publique est menée en 1934 dans le cadre des travaux de cette commission. Les solutions proposées pour accélérer l'électrification des campagnes sont de plusieurs ordres: les plus radicales demandent l'étatisation, d'autres se contentent de la municipalisation et d'autres encore s'en remettent aux coopératives soutenues par le gouvernement.

Dans leur rapport (janvier 1935), les commissaires optent pour la création de coopératives rurales d'électricité financées en partie par l'État, plutôt que pour une prise en charge gouvernementale directe. Ils écrivent: "L'organisation de coopératives qui désirent se former dans certains endroits dans le but de construire des lignes pour se relier aux grands réseaux devrait être encouragée par le gouvernement, et une partie des coûts de construction devrait être payée à titre de subvention à l'agriculture ${ }^{34}$.» Passant outre aux revendications exprimées par une portion significative des représentants du monde rural, à aucun moment les commissaires ne proposent que l'État construise lui-même les lignes rurales. Ils suggèrent plutôt la formation d'un organisme gouvernemental qui aurait entière juridiction sur les firmes d'électricité et qui remplacerait la Commission des services publics, qualifiée d'inefficace.

À la suite du rapport de la Commission Lapointe, le gouvernement Taschereau adoptera trois nouvelles lois relatives à la réglementation de l'industrie électrique et à la municipalisation de l'électricité ${ }^{35}$. Les recommandations des commissaires, particulièrement au sujet des coopératives, ne seront toutefois pas appliquées. L'unique mesure touchant l'électrification rurale est une subvention de $50 \%$ des coûts de construction des lignes rurales ${ }^{36}$. Seules les municipalités possédant, ou désirant établir, leur propre système de distribution sont admissibles. Cet article de la Loi de la municipalisation ne sera jamais utilisé. En définitive, la législation de 1935 n'apportera que des changements mineurs qui ne pourront guère accélérer l'électrification rurale du Québec.

34. Ibid., 27.

35. Québec (province) SDQ: Loi créant la Commission de l'électricité (25-26 Geo. V, 1935, chapitre 24); Loi concernant la municipalisation de l'électricité (25-26 Geo. V, 1935, chapitre 49); Loi relative à l'exploitation des chutes et des rapides situés sur les cours d'eau du domaine public et à leur concession à l'avenir (25-26 Geo. V, 1935, chapitre 23).

36. "[...] le trésorier de la province peut payer à toute municipalité une somme n'excédant pas $50 \%$ du coût capital de la construction et de l'établissement, dans ou pour le service de toute municipalité rurale, des lignes, câbles de transmission primaire, des transformateurs, des compteurs et des lignes secondaires de service électrique [...]" Québec (province) SDQ : Loi concernant la municipalisation de l'électricité (25-26 Geo. V, 1935, chapitre 49, article 19). 
Vraisemblablement destinées à apaiser l'opinion publique, la Commission Lapointe, puis les nouvelles mesures législatives, n'ont pas apporté au gouvernement Taschereau les résultats escomptés. Les intervenants du monde agricole sont insatisfaits et, dès l'adoption des lois, ils reprendront leurs doléances auprès de l'État. Au cours des dix années qui suivront, les études consacrées au "problème de l'électrification rurale» se multiplieront ${ }^{37}$. Mais les nouveaux organismes de l'État ne seront pas en reste cette fois, eux qui consacreront désormais une part importante de leur travail au problème de «l'électricité rurale».

Le premier d'entre eux, la Commission de l'électricité, met à son tour en évidence la lenteur de la diffusion du service électrique à la campagne: «La Commission conçoit qu'en cette matière d'électrification rurale, il reste beaucoup à faire ${ }^{38}$. " Elle n'aura cependant pas le temps d'agir, car après deux ans d'existence et l'arrivée au pouvoir de l'Union nationale en 1936, elle sera remplacée par la Régie provinciale de l'électricité.

Avec ses assises fortement rurales, ce nouveau gouvernement avait suscité des espoirs dans les milieux agricoles. Bien que Duplessis ait appuyé le principe des coopératives d'électricité, pendant sa campagne électorale, cette formule ne sera pas appliquée. La législation de $1937^{39}$ reconduit le même principe que celle de 1935 : le recours à la subvention. Celle-ci passe alors de $50 \%$ à $55 \%$, couvrant les coûts d'établissement d'un réseau municipal pour les localités rurales. À l'instar de la disposition de 1935, cette mesure soulèvera peu d'enthousiasme chez les dirigeants des municipalités ${ }^{40}$, lesquelles, depuis la formation des réseaux

37. À ce sujet, Albert Rioux (président de l'UCC de 1932 à 1936) est prolifique. C'est plus d'une quarantaine d'articles et de causeries qu'il publiera entre 1930 et 1944. Par exemple: « $\mathrm{A}$ propos d'électrification rurale", La Terre de chez nous, 8,7 (13 novembre 1935); "Pourquoi et comment étatiser?", Relations, 21 (septembre 1942): 259-261 ; "L'électrification des campagnes ", Relations, 21 (septembre 1942): 227-230; "L'électrification rurale et les compagnies privées", L'Action catholique, (11 février 1944). Sa thèse de doctorat porte également sur le thème: Albert Rioux, L'électrification rurale du Québec, op. cit.

38. Québec, Commission de l'électricité, Second Rapport pour l'exercice terminé le 30 juin 1937, Documents de la session (DSQ), 1940, $\mathrm{n}^{\circ} 35,19$.

39. Québec (province) SDQ: Loi autorisant toutes les corporations municipales à municipaliser l'électricité (1 Geo. VI, 1937, chapitre 26); Loi instituant la Régie provinciale de l'électricité (1 Geo. VI, 1937, chapitre 25) et Loi établissant et assurant la concurrence de l'État relativement aux ressources hydro-électriques (1 Geo. VI, 1937, chapitre 24). L'article concernant les subventions aux municipalités rurales ne figure pas dans la loi de la municipalisation mais est plutôt "enfoui " dans celle du Syndicat national de l'électricité (1 Geo. VI, 1937, chapitre 24, article 47).

40. Seulement quelques municipalités semblent s'être prévalues de ces subventions. Dans le rapport de la Régie de 1941, il est fait mention de trois "projets" soumis dans le cadre de cette 
régionaux, n'assument que rarement le service de l'électricité. L'ère de la production à grande échelle d'électricité exclut de plus en plus les municipalités.

Comme leurs prédécesseurs, les membres de la nouvelle Régie provinciale de l'électricité se disent conscients des difficultés que pose l'électrification des campagnes ${ }^{41}$. Mais l'organisme choisit, lui aussi, de ne pas contraindre les distributeurs à construire des lignes rurales en deçà d'un certain seuil de rentabilité. On considère généralement qu'un intérêt de $6 \%$ sur le capital investi dans la construction d'un tronçon de ligne rurale est justifiable ${ }^{42}$. Quoique le retour des libéraux au pouvoir en 1939 entraîne une fois encore des modifications législatives ${ }^{43}$, ce critère demeurera presque inchangé jusqu'en 1945.

La Régie des services publics, mise sur pied en 1940, se distingue néanmoins des commissions précédentes par le sentiment d'urgence ressenti : elle tient à préparer un plan d'électrification rurale pour l'aprèsguerre. Ses responsables admettent, comme plusieurs autres intervenants, que l'aide financière de l'État est nécessaire à l'électrification des zones rurales. Un véritable consensus se dégage alors dans les milieux intéressés quant à l'urgence d'accélérer le processus, avec l'espoir de stimuler ainsi la modernisation agricole du Québec. D’ailleurs, le monde agricole ne réclame plus seulement l'extension des réseaux de distribution à la campagne mais aussi le droit pour tous les cultivateurs de bénéficier des technologies reliées à l'électricité afin, notamment, d'accroître la productivité des exploitations.

loi. Dans Québec (province), Régie des services publics, Second rapport. Du 1 avril 1941 au 31 mars 1942, 30. Nous nous proposons d'approfondir ce sujet lors de recherches ultérieures.

41. Selon Y. Tremblay, «la Régie provinciale de l'électricité a amorcé une réorientation de sa politique. Pour la première fois, il a été décidé de faire subventionner par une catégorie d'abonnés (les citadins) les travaux d'extension du service rural. Mais la guerre, et la pénurie de matériaux qu'elle a entraînée, ont empêché la concrétisation de ce changement d'attitude.» Dans Y. Tremblay, "Entre le privé et le public... ", loc. cit., 178. Nous pourrions ajouter que le retour des libéraux au pouvoir en 1939 et l'abolition de la Régie provinciale ont quelque peu chamboulé ces plans.

42. Afin de déterminer si la "construction d'une ligne est ou n'est pas économique [...] la Régie a fixé, vers $1937-38$ à $6 \%$, et en 1944 entre $5 \%$ et $6 \%$, l'intérêt auquel le distributeur avait droit sur le capital placé [...]». En 1945, parce que les taux d'intérêts sont moins élevés, elle modifie légèrement ce critère. Il se situe maintenant entre $4,5 \%$ et $5 \%$. Dans Correspondance et rapports sur les coûts de construction de lignes rurales, $\mathrm{AHQ}$, fonds $\mathrm{P} 3$, document $\mathrm{n}^{\circ} \mathrm{P} 3 / 223$. Concrètement, cela équivaut à un minimum de dix clients par mille de ligne.

43. Québec (province) SDQ: Loi relative à l'aménagement de certaines chutes d'eau (IV Geo. VI, 1940, chapitre 23); Loi du contrôle de la force motrice en temps de guerre (IV Geo. VI, 1940, chapitre 12); Loi relative à l'exploitation des pouvoirs d'eau du domaine public (IV Geo. VI, 1940, chapitre 22) et Loi instituant la Régie des services publics (IV Geo. VI, 1940, chapitre 11). 
Il reste que rien de bien concret n'a encore été fait en 1944. En dépit de nombreuses études et de la gravité de la situation, l'État n'est toujours pas disposé à forcer les compagnies privées à électrifier les zones rurales et il hésite toujours à financer directement la construction de réseaux ruraux. La mobilisation de l'appareil productif pour les fins de la guerre n'est pas propice, il est vrai, à des investissements massifs dans l'électrification rurale.

\section{VERS L'ELECTRIFICATION MASSIVE DU MONDE RURAL}

Globalement, les marchés de l'électricité au Québec subissent une mutation profonde après la guerre. Contrairement à la situation qui prévalait durant les premières décennies du siècle, désormais ce ne sont plus les entreprises industrielles qui sont les principales responsables de la croissance des revenus des distributeurs, mais les consommateurs d'énergie en basse tension (les commerces et les domiciles, notamment $)^{44}$. Les exploitations agricoles participent à cette montée du marché des petits consommateurs. La modernisation progressive des fermes entraîne en effet une utilisation accrue de l'énergie électrique. De plus, les infrastructures de base des réseaux sont dorénavant en place, diminuant d'autant l'investissement requis pour desservir les fermes non encore branchées. Pressés par la menace coopérative, les grands distributeurs régionaux du secteur privé accélèrent l'électrification des campagnes.

Sur le plan politique, après une tentative ratée d'instauration d'un vaste programme d'électrification rurale sous l'égide de l'État, la formule coopérative sera finalement retenue. Malgré cette mise à l'écart de l'intervention directe de l'État, une conjonction de facteurs favorisera l'électrification accélérée des campagnes après 1945. Au sortir de la guerre et une dizaine d'années durant, la plupart des intervenants dans le secteur de la production et de la distribution d'électricité au Québec consentent enfin à investir massivement dans l'électrification des campagnes.

\section{La première étatisation}

L'élection d'un gouvernement libéral en 1939 annonçait des changements majeurs dans le domaine de l'électricité au Québec ${ }^{45}$. Pendant les

44. Claude Bellavance, Roger Levasseur et Yvan Rousseau, loc. cit., et J. H. Dales, op. cit.

45. Le nouveau premier ministre, Adélard Godbout, est agronome de formation. C'est à ce titre d'ailleurs qu'il est intervenu publiquement, dès 1930, en faveur de l'électrification rurale. 
années de guerre, le gouvernement Godbout a préparé une grande réforme dont l'objectif est de confier à l'État une responsabilité directe dans le secteur électrique. En avril 1944, il crée Hydro-Québec, qui reprend les actifs de Montreal Light, Heat $\&$ Power (MLHP) et de ses filiales.

La création d'une entreprise publique marque une véritable rupture quant au mode d'action privilégié jusqu'ici par les différents gouvernements pour réglementer l'industrie hydro-électrique québécoise et en orienter le développement. L'étatisation de MLHP découle, on le sait, d'une enquête effectuée par la Commission de l'électricité (et poursuivie par ses successeurs) qui avait révélé les pratiques financières douteuses de l'entreprise montréalaise. Ce que l'historiographie a moins retenu cependant, c'est que le gouvernement Godbout avait choisi de doter la Commission hydro-électrique de Québec (Hydro-Québec) de pouvoirs très étendus en matière d'électrification rurale. C'est sous cet angle que nous analysons brièvement ici l'impact de la création d'Hydro-Québec.

Toute une section du mandat de la nouvelle société d'État concerne l'électrification rurale. En effet, sa loi constitutive ${ }^{46}$ prévoit une forte injection de deniers publics destinés à l'érection de réseaux ruraux d'électricité. Un budget de dix millions est prévu pour desservir les municipalités rurales. En outre, une somme supplémentaire de un million de dollars doit servir à aider directement les agriculteurs désireux de faire les installations nécessaires pour bénéficier de l'électricité. A. Godbout estime qu'en cinq ans, l'ensemble du Québec pourrait être électrifié ${ }^{47}$.

La mise sur pied d'un programme d'électrification rurale financé par l'État répond aux demandes exprimées depuis le début des années 1930. Lors de l'enquête de la Commission Lapointe, ils étaient nombreux à réclamer l'adoption d'une politique inspirée du régime ontarien. Les dispositions prévues par la loi d'Hydro-Québec ressemblent, sur bien des points, à ce modèle: création d'une entreprise publique d'électricité, construction par l'État d'un réseau de distribution rurale et possibilité pour les cultivateurs d'emprunter afin de raccorder leur exploitation au

"Quelques notes sur notre dernier congrès [de l'UCC à Québec, 1930]", La Terre de chez nous, 3,2 (26 novembre 1930): 28. À noter qu'il se prononce alors contre l'étatisation bien qu'il souhaite que les fermes québécoises disposent de l'électricité le plus rapidement possible.

46. Québec (province) SDQ, Loi établissant la Commission hydro-électrique de Québec. (8 Geo. VI, 1944, chapitre 22). C'est la section VII qui régit l'électrification rurale.

47. A. Godbout, session du 28 mars 1944, dans Jocelyn Saint-Pierre, dir., Hydro-Québec, Débats parlementaires, Loi 17-1944 (Québec, Bibliothèque de l'Assemblée nationale, 1994), 25. 
réseau ${ }^{48}$. Sans doute peut-on y voir l'impact des études réalisées par les experts de la Commission et de la Régie de l'électricité, et qui prenaient le système ontarien pour modèle.

Nous ne disposons pas de témoignages directs nous permettant de mesurer l'accueil réservé à la société d'État dans les milieux agricoles ${ }^{49}$. Cependant, le nombre élevé de requêtes acheminées à Hydro-Québec dans un court laps de temps laisse croire que plusieurs ruraux fondent beaucoup d'espoir dans la création de cette entreprise publique, qui prévoit un important soutien à l'électrification rurale ${ }^{50}$.

Les pouvoirs conférés à Hydro-Québec pour électrifier les campagnes ne seront toutefois jamais utilisés. Le retour de Maurice Duplessis à la tête du Québec à l'automne de 1944 reléguera aux oubliettes le projet du gouvernement d'Adélard Godbout de poursuivre l'électrification du monde rural par l'entremise d'une société d'État ${ }^{51}$.

\section{À la recherche d'un compromis: l'approche duplessiste}

Bien que son parti trouve sa principale base électorale en milieu rural, Maurice Duplessis s'oppose au principe de l'intervention de l'État. Partisan du laisser-faire en matière économique ${ }^{52}$, il semble ne pas

48. L'article 22 offre une autre similitude intéressante. Hydro-Québec «doit établir le tarif applicable à chaque catégorie d'usagers suivant le coût réel du service fourni à cette catégorie en autant que cela est pratique». Ce principe rappelle la politique d'Hydro-Ontario surnommée Power at cost. À ce sujet, voir K. R. Fleming, op. cit.

49. Mais les régisseurs de la Régie des services publics se montrent confiants: "La création de la Commission Hydro-électrique dont le projet de loi est déposé devant l'Assemblée marquera le début d'une ère nouvelle [...]. La subvention directe de l'État et son introduction dans le domaine de la distribution constituent le prochain essai dans le sens de l'électrification des campagnes et la classe agricole ne manquera certes pas d'exploiter cette nouvelle formule d'assistance.) Dans Régie des services publics, Quatrième rapport, 1944, 29.

50. Entre le 14 avril 1944 (date de sa création) et le 31 décembre 1944, Hydro-Québec a reçu 145 requêtes, dont 108 lui ont été directement adressées, les autres ayant été transmises par la Régie des services publics. Premier rapport annuel de la Commission Hydro-électrique pour l'année se terminant le 31 décembre 1944.

51. La loi d'Hydro-Québec de 1964 (SRQ, 1964, vol. 2, chapitre 86, articles 43 à 47) contient toujours cette section sur l'électrification rurale. Mais les rapports annuels d'Hydro-Québec n'offrent aucune considération sur le sujet et nous laissent croire que les pouvoirs donnés par cet article n'ont jamais été utilisés. Seul le premier rapport (1944) contient des informations. On y trouve une dépense pour l'électrification rurale de $15340 \$$ inscrite dans le bilan consolidé d'Hydro-Québec pour l'année 1944. Cette dépense découle d'études préliminaires de l'électrification de certaines régions rurales effectuées par la société d'État au cours des neuf premiers mois de son existence. Elle constitue la seule mention de ce genre dans tous les bilans financiers d'Hydro-Québec entre 1944 et 1961. Premier rapport annuel, Commission Hydro-électrique, op. cit.

52. Sur la politique économique de Duplessis, consulter Gérard Boismenu, Le duplessisme: politique économique et rapports de force, 1944-1960 (Montréal, Presses de l'Université de Montréal, 1981). 
vouloir inquiéter davantage les compagnies d'électricité, déjà préoccupées par l'étatisation de l'une d'entre elles. Il préfère mettre sur pied l'Office de l'électrification rurale, dont le principal mandat sera d'appuyer la formation de coopératives rurales d'électricité.

La formule coopérative est d'ailleurs défendue par une partie des milieux agricoles depuis les années 1930. C'est l'ancien président de l'UCC, Albert Rioux, qui sera chargé, avec l'aide de deux avocats du gouvernement, de préparer le projet de loi $^{53}$. La Loi pour favoriser l'électrification rurale par l'entremise des coopératives d'électricité ${ }^{54}$ est sanctionnée le 24 mai 1945. Ainsi est institué l'Office de l'électrification rurale (OER). La création de cet organisme doit être interprétée comme un compromis ayant pour objectif de réduire au minimum l'intervention de l'État, puisque ce sont les collectivités locales qui organiseront les coopératives.

L'Office est chargé de superviser la fondation puis le fonctionnement de coopératives rurales d'électricité. L'aide technique, juridique ou d'ingénierie dont peuvent avoir besoin les coopératives est également du ressort de l'OER. Toute coopérative formée pour desservir une portion du territoire rural québécois est éligible au financement public. L'Office gère l'aide gouvernementale ${ }^{55}$. Il consent des prêts aux coopératives pouvant atteindre $75 \%$ du coût d'aménagement des réseaux ${ }^{56}$. Quant aux coopératives, elles s'engagent à rembourser annuellement $3 \%$ du montant prêté, pendant 30 ans. La subvention de l'État ne représente donc au plus que $10 \%$ de ces sommes. La loi prévoit aussi un système de prêts afin d'aider les coopérants à doter leur exploitation des installations requises pour recevoir l'électricité.

Les coopératives rurales ont sensiblement les mêmes pouvoirs que les distributeurs privés d'électricité, c'est-à-dire qu'elles sont habilitées à construire, louer, posséder, administrer des barrages, des usines, des centrales électriques, des lignes de transmission primaire et secondaire, des lignes de distribution ainsi que toutes machineries s'y rapportant (article 32a). De plus, elles peuvent, avec l'approbation de l'OER,

53. Sur Albert Rioux et la mise en place des coopératives, voir Yves Tremblay, «Albert Rioux et la création de l'Office de l'électrification rurale", op. cit.

54. Québec (province) SDQ, Loi pour favoriser l'électrification par l'entremise de coopératives d'électricité ou Loi de l'électrification rurale (9 Geo. VI, 1945, chapitre 48).

55. Le montant total que l'Office est autorisé à dépenser est d'abord fixé à 12 millions de dollars en 1945 puis graduellement augmenté pour atteindre 35 millions en 1955.

56. À partir de 1948, le financement peut atteindre $85 \%$ lorsque la densité moyenne des usagers de la coopérative est inférieure à six par mille de réseau. Québec (province) SDQ, Loi modifiant la Loi de l'électrification rurale (12 Geo. VI, 1948, chapitre 40). 
"acquérir par voie d'expropriation les biens meubles et immeubles énumérés au paragraphe $a$ du présent $\operatorname{article~}^{57}$ ». Enfin, le réseau des coopératives doit être situé dans l'une des sept zones d'électrification définies par l'Office: 1) Abitibi-Témiscamingue, 2) Laurentides, 3) Cantons-de-l'Est, 4) Golfe du Saint-Laurent, 5) Lac Saint-Jean, 6) BasSaint-Laurent et 7) Gaspésie.

Les zones ainsi délimitées par l'OER empiètent parfois sur les aires d'influence des grandes compagnies privées d'électricité. C'est le cas, notamment, dans les Cantons-de-l'Est (Southern Canada Power) et en Gaspésie, de même qu'au Bas-Saint-Laurent (Compagnie de Pouvoir du Bas-Saint-Laurent). Dans les faits, peu de distributeurs sont vraiment à l'abri de la concurrence potentielle de coopératives d'électricité sur leur territoire ${ }^{58}$. L'instauration du régime des coopératives, conjuguée à la nationalisation de Montreal Light, Heat $\&$ Power, mettent fin à 50 ans d'exploitation privée de l'électricité au Québec. Comme nous le verrons maintenant, l'impact de ces changements est immédiat. Non seulement plusieurs coopératives sont rapidement fondées, mais les entreprises d'électricité, qui appréhendent désormais la concurrence de coopératives sur leur territoire, construisent avec beaucoup de célérité de nouvelles lignes rurales.

\section{L'électrification accélérée du monde rural}

Les années d'après-guerre sont caractérisées par l'électrification massive des exploitations agricoles. En six ans seulement, de 1945 à 1951, la proportion d'exploitations électrifiées a plus que doublé, passant de 28 à $67 \%{ }^{59}$. Au total, le pourcentage d'exploitations électrifiées sera multiplié par 3,5 au cours des années 1945-1961. En 1961, $97 \%$ des fermes québécoises disposent de l'électricité (figure $2{ }^{60}$.

L'électrification accélérée du monde rural s'effectue cependant sans que l'État cherche à lui imprimer une direction. En effet, chacun des

57. Il ne semble pas que ces pouvoirs aient été largement utilisés.

58. Des coopératives seront fondées à la grandeur du Québec. Plusieurs d'entre elles ne seront cependant jamais actives.

59. Ce phénomène n'est toutefois pas particulier au Québec, comme le souligne K. R. Fleming au sujet de l'Ontario: "The most notable feature of the post-war period was the speed at which the rural network was completed. » Keith R. Fleming, op. cit., 247.

60. Les pourcentages d'électrification rurale du Québec ne sont malheureusement pas disponibles sur une base annuelle pour toute la période : il existe des carences de 1947 à 1951, en 1954 et en 1955). Les données manquantes ont donc été calculées à partir du gain moyen des années précédentes. 
FIGURE 2

\section{La progression de l'électrification rurale au Québec, de 1930 à 1961}

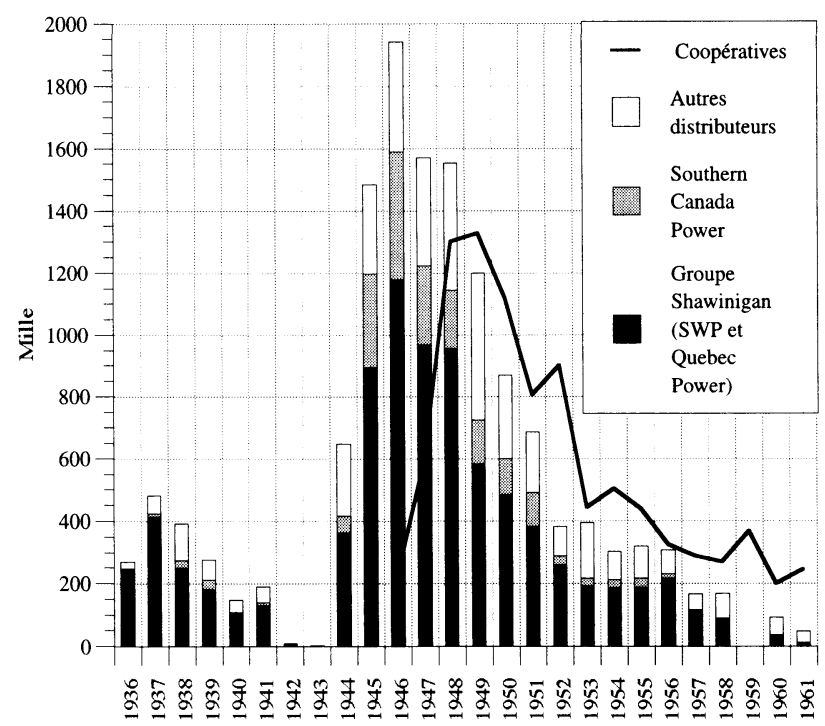

Compilation de l'auteure. Sources : AHQ, P3/235 et Rapports annuels de l'OER.

Cette figure inclut toutes les lignes rurales, y compris celles qui sont construites dans les villages et, à partir de 1958,

ces chiffres incluent, pour Gatineau et SWP surtout, des clients saisonniers. Autres distributeurs regroupe Gatineau Power, La compagnie électrique du Saguenay, la Compagnie de Pouvoir du Bas-Saint-Laurent et tous les autres petits distributeurs.

organismes gouvernementaux responsables a élaboré un programme dans sa sphère d'intervention: la Régie provinciale de l'électricité auprès des compagnies privées, l'Office de l'électrification rurale auprès des coopératives et Hydro-Québec dans son territoire circonscrit de la région montréalaise. Quant aux municipalités qui assument la distribution de l'électricité, telle Sherbrooke, elles ne sont l'objet d'aucune loi particulière ${ }^{61}$. Tout indique qu'une coordination minimale était pratiquée entre les diverses instances agissant dans la poursuite de l'électrification rurale ${ }^{62}$.

61. Les services municipaux ne relèvent pas de l'Office d'électrification rurale, et ni la loi de la Régie provinciale de l'électricité ni celle d'Hydro-Québec ne nous permettent de croire, en ce qui a trait à l'électrification rurale, qu'ils sont sous leur juridiction.

62. Un bon exemple du faible degré d'intégration des politiques gouvernementales est fourni par l'ingénieur en chef de la Régie, J.-A. Beauchemin, en 1946. Dans une lettre adressée au président de la Canadian Electrical Association qui s'informait du «Program of Quebec for rural electrification in the immediate future ", il explique le fonctionnement de l'électrification rurale 
En dépit de l'absence de véritable planification en matière de construction de lignes rurales, la Régie et l'OER collaborent tout de même dans certaines régions afin d'établir un modus vivendi entre les coopératives et les compagnies privées œuvrant dans des zones contiguës. En Abitibi et dans les Cantons-de-l'Est, de même qu'au Bas-Saint-Laurent, les distributeurs privés doivent composer avec plusieurs coopératives très actives.

\section{Les nouvelles modalités de l'électrification des campagnes}

La mise en perspective des conditions sociopolitiques de l'électrification rurale nous a permis de cerner un aspect important du processus. Cependant, la progression des réseaux d'électricité en milieu rural obéit également à des contraintes économiques et techniques. La proximité des centres urbains, la densité des populations à électrifier, de même que la volonté des compagnies privées de prolonger leurs lignes jouent un rôle majeur. À ce chapitre, la fin de la Seconde Guerre mondiale marque une rupture: les facteurs économiques invoqués depuis 15 ans par les distributeurs pour justifier leur peu d'intérêt envers les zones rurales sont relégués au second plan. Ils ont désormais un territoire, et une image, à sauvegarder. Nous verrons maintenant que le mode de diffusion de l'électricité en milieu rural ${ }^{63}$ change complètement après 1945.

L'effort conjugué des distributeurs privés et des coopératives entraînera, à partir de 1945, une forte augmentation de la proportion d'exploitations agricoles électrifiées (figure 2). Aussi saisissants soient-ils, ces progrès cachent cette fois encore de fortes disparités régionales.

Comme l'indique la cartographie des données rassemblées par comtés en 1951 (carte 2), la situation est bien différente de celle de 1931 (carte 1), car un plus petit nombre de comtés affiche un pourcentage d'électri-

au Québec: "Farms electrification is achieved in this Province through four types of organisations: 1 - Power companies. [...]. 2 -Hydro-Quebec. A government-owned power and distribution system. [...]. 3 - Municipal systems: Sherbrooke, Joliette, Grand-Mère, Rivière-duLoup, Jonquière, Bagotville, Magog, [...] etc. 4-Rural Electrification Bureau. A Provincial government commission to promote electrification through cooperatives. [...] Each of these organisations has its own construction program and you might get in touch with them for more securate information." Dans Correspondance et rapports sur l'électrification rurale, AHQ, fonds $\mathrm{P}$, document $n^{\circ} \mathrm{P} 3 / 215$. C'est nous qui soulignons. L'analyse du fonctionnement des différentes régies et de leurs relations avec les autres organismes reste à faire.

63. À noter que nous n'abordons pas cette question en proposant un modèle de diffusion spatiale, au sens donné à ce terme par la géographie des modélisations. À ce sujet, voir notamment: Thérèse Saint-Julien, "Diffusion spatiale", dans Antoine Bailly et al., Les concepts de la géographie humaine (Paris, Masson, 1991), 559-581. 
fication inférieur à la moyenne québécoise (67\%). Ils n'en paraissent que plus marginaux encore. La persistance de ces disparités illustre la double dynamique qui, après 1945, oppose le centre agricole du Québec à ses marges. Il y a, d'abord, les régions massivement électrifiées entre 1945 et 1951. Bagot (on y est passé de $9 \%$ des exploitations agricoles électrifiées en 1931, à $88 \%$ en 1951), Dorchester (de $10 \%$ à $85 \%$ ) et Iberville (de $10 \%$ à $91 \%$ ) sont, à cet égard, particulièrement représentatifs. Ces comtés agricoles fortement électrifiés font presque tous partie des basses terres du Saint-Laurent et ils sont, pour la plupart, desservis par des distributeurs privés d'électricité. Les autres, moins nombreux, devront au contraire patienter après 1951 pour bénéficier d'un réseau électrique convenable. L'électrification de ces comtés, majoritairement situés en périphérie, est essentiellement conduite par des coopératives rurales ${ }^{64}$.

Ce sont donc les régions alimentées par les entreprises privées qui ont disposé le plus rapidement de l'électricité. Il ne s'agit cependant pas d'une règle absolue. Prenons par exemple le couloir Mégantic-Compton, qui reste sous-électrifié. Il est pourtant desservi par la plus grande entreprise d'électricité du Québec, Shawinigan Water \& Power. L'absence de centres industriels d'importance, la topographie accidentée, le potentiel agricole souvent limité et une faible densité de peuplement peuvent expliquer son bas niveau d'électrification. SWP électrifiera Mégantic et Wolfe après 1945, alors que Compton sera en partie occupé par les coopératives fondées dans cette zone.

La sous-électrification de l'Outaouais et du nord-ouest du Québec persiste également. En Outaouais, deux conditions favorables au prolongement des lignes rurales sont pourtant réunies: la présence de centres urbains (Hull et Gatineau) et un distributeur d'envergure régionale, Gatineau Power. Ce dernier est un important producteur d'électricité, mais son existence répond à des besoins essentiellement industriels, au détrimenet de la clientèle domestique. L'entreprise n'a même aucun programme d'électrification rurale, contrairement à la majorité des grands distributeurs. Par ailleurs, aucun distributeur d'importance n'est présent dans le nord-ouest de la province. On y trouve bien des firmes de petite taille, mais elles demeurent cantonnées dans les aires villageoises et ne disposent pas, généralement, des moyens techniques et

64. Le Bas-Saint-Laurent est un cas particulier, car les coopératives y seront en fait exploitées par la Compagnie de pouvoir du Bas Saint-Laurent. Voir Yves Tremblay, "L'Office et les problèmes particuliers du Bas-Saint-Laurent ", Histoire sociale et technique de l'électrification..., op. cit., 342-358. 
CARTE 2

\section{Les exploitations agricoles électrifiées au Québec,} par comtés, en 1951

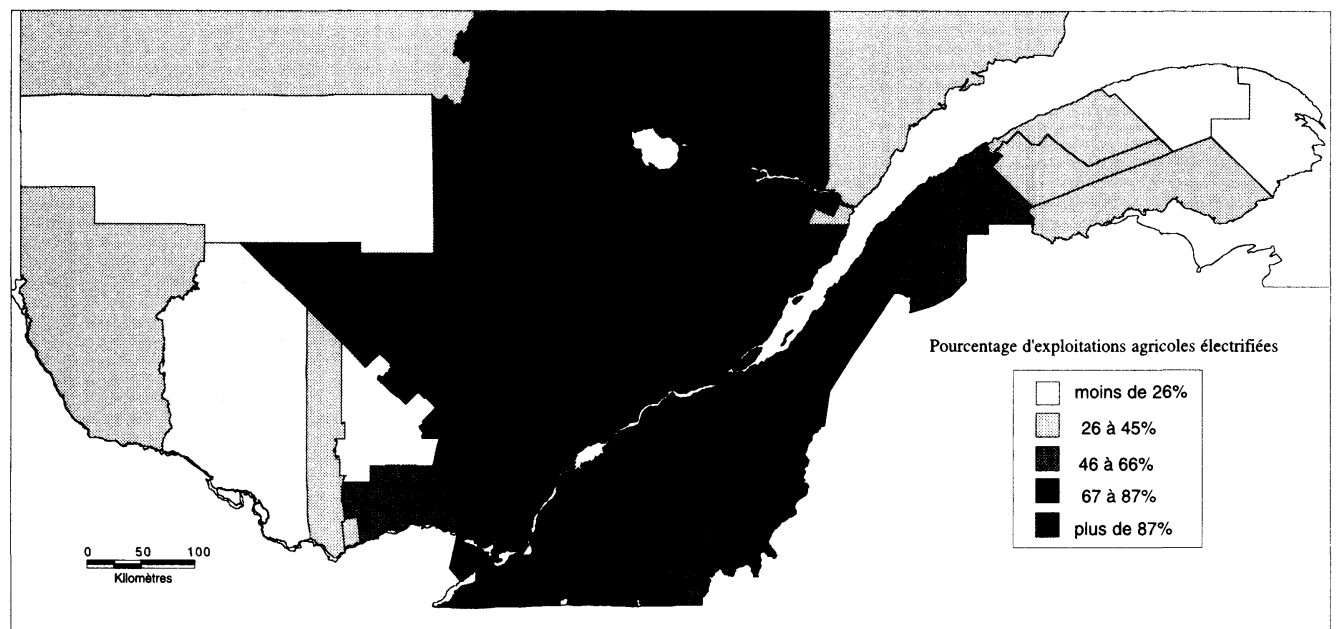

Source: Recensement du Canada, 1951.

financiers pour étendre leurs réseaux. Ce n'est donc pas un hasard si cette région sera parmi les plus actives du mouvement coopératif de l'après-guerre. Dès 1947, le réseau de la Coopérative d'électricité du Témiscamingue, par exemple, se déploie sur plus de 240 milles.

À l'échelle québécoise, les coopératives, formées à partir de 1945, ne commenceront l'érection de leurs réseaux qu'après 1946 (figure 3). Les grands distributeurs, eux, aménagent un nombre impressionnant de lignes rurales entre 1944 et 1951 . Ce décalage de quelques années entre la plus forte période de construction des coopératives et celle des compagnies $^{65}$ peut expliquer, du moins en partie, la démarcation entre la partie centrale du territoire québécois et ses marges. C'est en 1946 (l'Office de l'électrification rurale est créé en 1945) qu'est atteint le nombre maximum de milles construits par les compagnies.

Cette figure inclut toutes les lignes rurales, y compris celles qui sont construites dans les villages et, à partir de 1958, ces chiffres incluent,

65. Il est difficile de déterminer quelle part occupent les systèmes municipalisés. Selon la Régie, ils représentaient $1 \%$ de la distribution rurale en 1943. Dans AHQ, fonds P3, document $\mathrm{n}^{\circ} \mathrm{P} 3 / 219$. Quant à Hydro-Québec, comme nous l'avons déjà mentionné, les considérations relatives à l'électrification rurale contenues dans son premier rapport sont absentes des rapports suivants. 
FIGURE 3

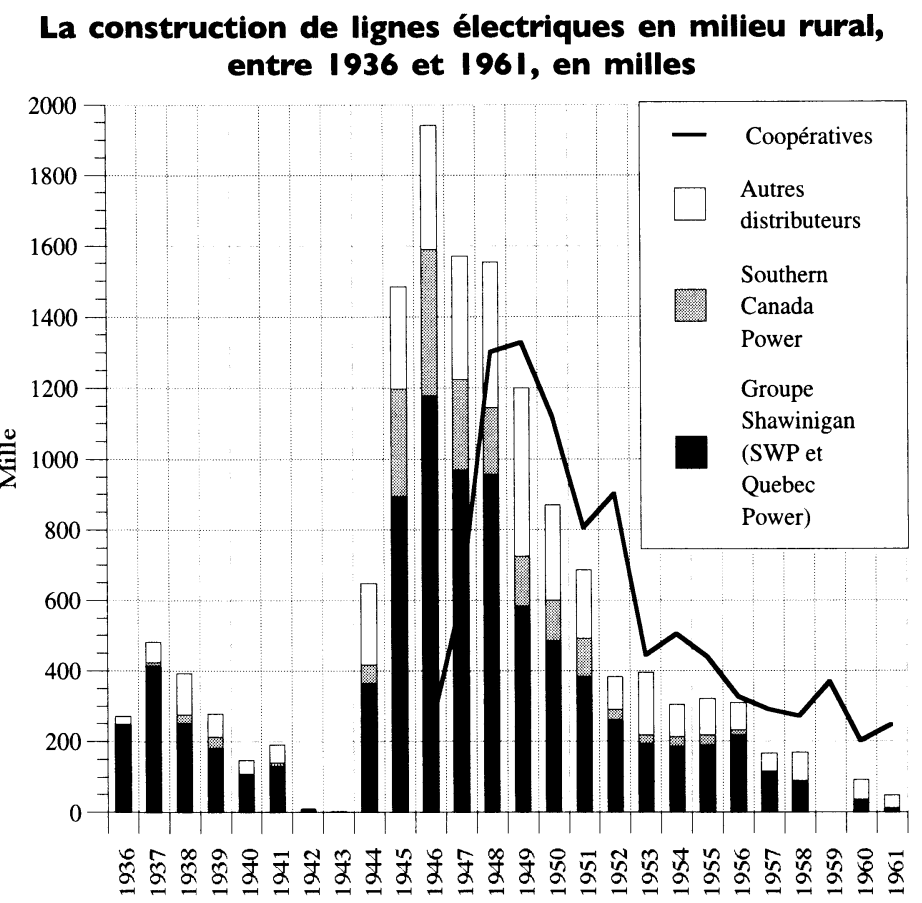

pour SWP et Gatineau surtout, des clients saisonniers. Autres distributeurs regroupe Gatineau Power, la Compagnie électrique du Saguenay, la Compagnie de Pouvoir du Bas-Saint-Laurent et tous les autres petits distributeurs.

La diffusion de l'électricité à l'échelle infrarégionale

L'influence de l'OER et des coopératives est perceptible même dans les régions où les compagnies privées sont omniprésentes. C'est le cas dans les comtés de Nicolet et de Yamaska que nous examinerons plus attentivement maintenant. Les sept coopératives qui s'y retrouvent ne construiront pas de lignes rurales, mais elles seront perçues, par SWP ou Southern Canada Power (SCP), comme des concurrentes, au même titre que les petites firmes d'électricité qui avaient été absorbées au cours des années 1910 et $1920^{66}$.

66. À l'échelle du Québec, au cours des premières décennies du siècle, les grandes entreprises d'électricité ont acquis la majeure partie des firmes locales. Voir Claude Bellavance, Shawinigan Water \& Power..., op. cit. Les comtés de Nicolet et de Yamaska n'ont pas échappé à ce processus d'intégration. Au sujet des entreprises d'électricité ayant œuvré dans les comtés de Nicolet et de Yamaska, voir Marie-Josée Dorion, op. cit., 59-64. 
Dans les paroisses où des coopératives ont été fondées ${ }^{67}$, SCP et SWP vont prolonger très rapidement leurs réseaux de distribution. Les comtés de Nicolet et de Yamaska seront rapidement électrifiés au cours des années d'après-guerre. Dans Nicolet, le pourcentage d'électrification rurale passe de $5,6 \%$ en 1931 à $80 \%$ en 1951. Dans Yamaska, la proportion grimpe de $8 \%$ à $83 \%$ pour les mêmes années ${ }^{68}$. Dès 1948 , quelques paroisses sont presque complètement électrifiées. Cette stratégie porte fruit, car aucune autre coopérative ne sera mise sur pied dans la région après 1947. Les compagnies privées du Centre du Québec étendent néanmoins avec empressement leurs réseaux ruraux même dans les localités où il n'existe aucune coopérative. La construction des principales lignes de transport et de distribution de l'électricité obéissait, avant la Seconde Guerre mondiale, à plusieurs facteurs tous interreliés dont ne faisaient pas, ou très peu, partie les réclamations des habitants de la région $^{69}$. Les considérations strictement économiques, c'est-à-dire la rentabilité de chacun des tronçons pris isolément ${ }^{70}$, primaient. La proximité des centres industriels et la localisation des lignes de transmission étaient également déterminantes.

En fait, la stratégie commerciale des distributeurs a eu un impact certain sur l'accessibilité au service électrique. Le réseau de transport de

67. Comté de Yamaska: La Coopérative régionale d'électricité de Saint-Guillaume-d'Upton incorporée en 1945, celle de Saint-Elphège (1946) et celle de La Visitation-de-Yamaska (1946). Comté de Nicolet: la coopérative de Gentilly incorporée en 1946, celle de la paroisse de SainteMonique (1946), celle de Sainte-Marie-de-Blandford (1946) et celle de Saint-Samuel-de-Horton (1946). Ces sept coopératives ne seront jamais actives. Cette situation n'est pas particulière aux comtés de Nicolet et de Yamaska. Dans l'ensemble du Québec, 193 coopératives ont été incorporées entre 1945 et 1963 ; seulement 58 (le maximum de 1960) d'entre elles seront actives. Historique de l'Office de l'électrification rurale, préparé par le secrétaire de l'Office, 21/09/1988, AHQ.

68. Selon les RDC.

69. Par exemple, Commission de l'électricité, Dossier de l'ordonnance n ${ }^{\circ} 554$. Paroisse de SaintPie-de-Guire, comté de Yamaska Vs SWP. Demande du conseil municipal de "faire passer l'électricité dans cette paroisse ", suivie d'une requête de 20 cultivateurs du $13^{\mathrm{e}}$ rang pour obtenir le service de l'électricité. Dans le Premier rapport annuel de la Régie de l'électricité de 1939, la décision est la suivante: "Les revenus provenant d'environ 25 intéressés ne justifient pas une mise de capital de 13000 \$. Requête refusée." La ligne sera érigée en 1946. Dans Programmes d'électrification rurale de SWP, document $\mathrm{n}^{\circ} \mathrm{P} 3 / 236$ et fonds de la CSPQ.

70. La Régie - comme les distributeurs - calcule la rentabilité intrinsèque de chacun des tronçons à construire et non pas celle de l'ensemble du système de distribution. Elle avait donc fixé à dix le nombre minimal d'usagers "par mille de ligne " pour parvenir au seuil de rentabilité. La densité des clients ruraux atteint toutefois difficilement un tel niveau : entre 1936 et 1946, dans Nicolet et Yamaska, la moyenne est de 7,2, selon nos calculs. AHQ, fonds P3, documents $\mathrm{n}^{\text {os }} 224,236$ et 237. 
la rive sud avait en effet été érigé en 1906 pour relier les entreprises d'amiante d'Asbestos et de Thetford Mines aux installations de SWP, au nord de Trois-Rivières. L'érection par celle-ci, en 1914-1916 (voir la carte, en annexe), d'une seconde ligne à haute tension en direction ouest, vers Sorel, répondait à ce même impératif.

Les postes d'arrivée des lignes de transport, aménagés à SainteAngèle, à Saint-Grégoire et à Pierreville, ont alors servi de base à SWP (et à ses filiales) pour quadriller de ses lignes de distribution tout le territoire contenu entre la rive du fleuve et la région de l'amiante. Des lignes de distribution ont été graduellement construites, à partir du réseau de transport, pour desservir les municipalités, de la plus rapprochée vers la suivante. Ce sont les villages qui ont d'abord reçu le service $^{71}$. Toutefois, les lignes de distribution ne couvraient souvent qu'une portion des villages desservis. La situation est encore plus frappante en dehors des limites villageoises. Car, si les compagnies ont prolongé les lignes villageoises vers les paroisses dès les années 1920, ces lignes restaient peu étendues et visaient essentiellement les rangs les plus peuplés situés autour du village.

Cette manière de faire a eu une incidence majeure sur la proportion d'exploitations agricoles électrifiées. On observe encore, effectivement, de grandes disparités parmi les paroisses desservies par le réseau de SWP en 1941 (carte 3). Le taux varie entre $4 \%$ et $66 \%$. L'étude détaillée $e^{72}$ révèle également la remarquable concentration géographique des exploitations agricoles qui disposent alors de l'électricité. Nous décelons dans cette situation le reflet des stratégies d'extension de réseau de SWP, car les municipalités les mieux desservies sont situées sur le parcours des premières lignes de transmission (voir la carte en annexe). Plusieurs paroisses éloignées des infrastructures de base sont au contraire faiblement électrifiées.

71. Nicolet, la seule ville de cet espace au caractère rural très affirmé, sera la première à en bénéficier et ce, dès 1909. Ce sont les villages les plus importants - Sainte-Angèle-de-Laval en 1914, Pierreville en 1915 et Baieville en 1916 - qui seront par la suite électrifiés. Plusieurs sources ont été recoupées afin de déterminer la séquence d'électrification des villages et paroisses de la région: Canada, Ministère du Revenu de l'intérieur, Rapports, états et statistiques des revenus de l'intérieur du Canada; Inspection des poids et mesures, du gaz et de l'éclairage électrique de 1900 à 1930; Canada, Département de l'intérieur, Central Electric Station in Canada, appendice Directory, 1918, 1919, 1922 et 1928; États financiers des compagnies en cause et ordonnances émises par les diverses instances de contrôle entre 1909 et 1944.

72. Nous avons des données précises pour 27 paroisses des comtés de Nicolet et de Yamaska, sur un total de 40 , ce qui représente $68 \%$ d'entre elles. 


\section{CARTE 3}

\section{Les exploitations agricoles électrifiées dans les comtés de Nicolet et de Yamaska, en 1941 (paroisses desservies par Shawinigan Water \& Power)}

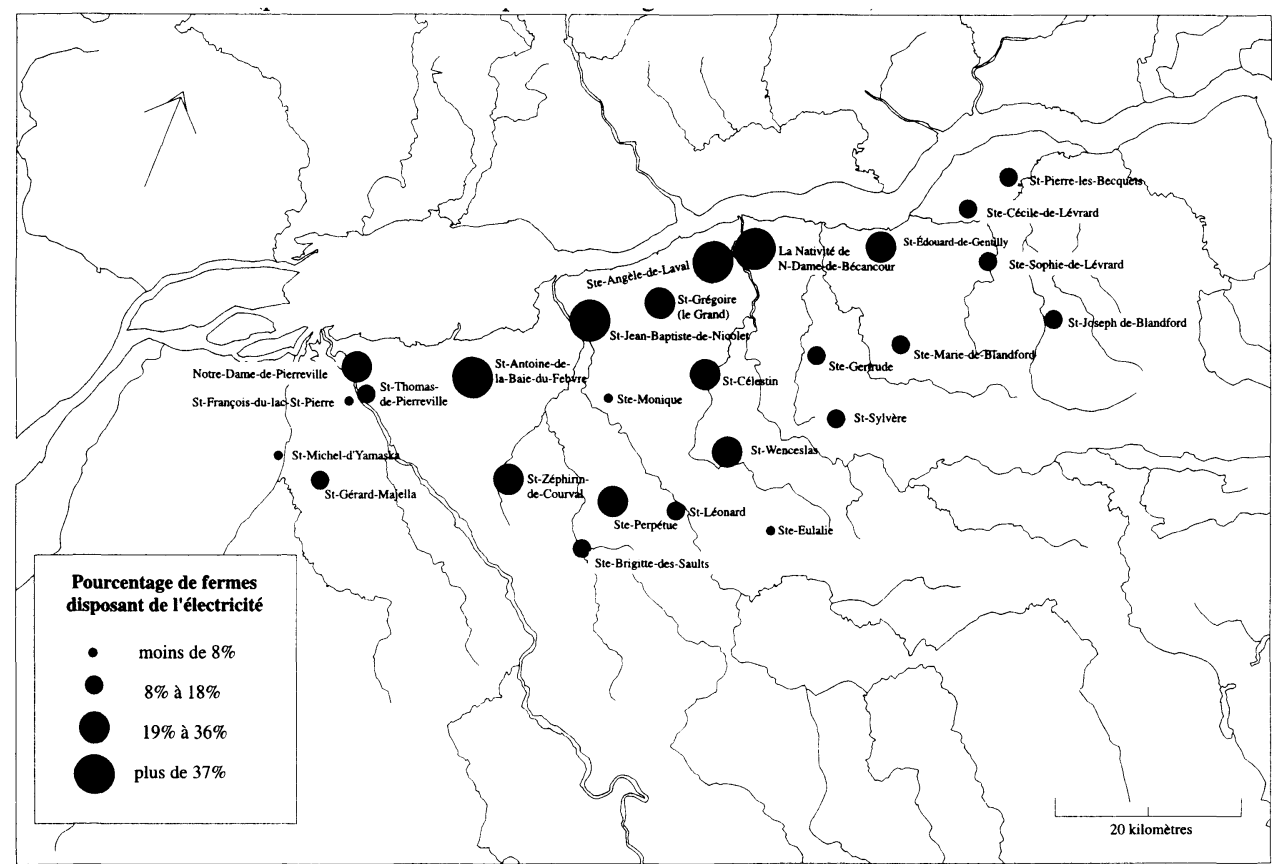

Sources : RDC et États financiers de SWP pour l'année 1941.

Ainsi, l'érection des réseaux avait suivi avant 1945 un même schéma de ramifications en reliant entre eux les points les plus denses de concentration de population. Il en va tout autrement après 1945. Le coût des infrastructures locales et régionales de transport d'énergie et celui des installations de distribution (comme les sous-stations) sont maintenant amortis grâce, notamment, aux revenus tirés des villes et des villages. Les lignes peuvent alors s'étendre vers les zones moins densément peuplées. Le mode d'extension du réseau rural n'est donc plus le même après 1945 , les nouvelles constructions apparaissant çà et là sur le territoire, sans lien apparent avec le degré de concentration des populations. La progression du réseau sous forme de quelques branches et de ramures, telle qu'elle s'est faite dans les années 1910-1944, a été remplacée par un développement autrement plus ramifié. L'éparpillement des 
nouvelles lignes vise, c'est évident, à répondre dans les meilleurs délais possibles aux revendications les plus pressantes. Plusieurs paroisses qui réclament depuis longtemps l'électrification de leurs rangs seront ainsi desservies entre 1946 et $1949^{73}$.

L'importance relative de la densité de l'habitat, élément clé de la distribution d'électricité jusqu'au milieu des années 1940, diminue. Pour les lignes rurales, le minimum de " 10 clients au mille " n'est plus systématiquement respecté. Après 1945, les distributeurs, SWP en tête, font abstraction de ce facteur pour un très grand nombre de nouvelles lignes rurales. Nous avons pu établir que les constructions effectuées par SWP dans les comtés de Nicolet et de Yamaska entre 1945 et 1955 affichent en effet un nombre de clients probables par mille qui oscille entre 2,5 et 10,1 (moyenne de 6,1 $1^{74}$ ). C'est après 1951 qu'on note une baisse importante du nombre d'usagers moyens par mille, signe que l'on accepte maintenant de relier des endroits où la densité est relativement faible ${ }^{75}$. On électrifie dorénavant des "bouts de rangs" et des zones considérées jusqu'ici comme "non rentables». Nous pouvons y voir un reflet de stratégies commerciales de longue portée. Les transformations de l'agriculture commandent l'utilisation de nombreux appareils électriques et les compagnies perçoivent dorénavant le monde rural comme un marché à développer, au même titre, sans doute, que le marché urbain des années 1920.

Quelques paroisses, pour la plupart situées loin des principaux points de concentration de la population et des infrastructures de base, devront

73. Un exemple représentatif: Régie de l'électricité, Dossier de l'ordonnance $n^{\circ} 646$, "SaintZéphirin-de-Courval: Requête reçue le 19 juillet 1937 portant 50 signatures, pour extension de service de l'électricité aux rangs Saint-François, Saint-Pierre, Sainte-Geneviève, Saint-Michel et Châtillon, sur une distance de 9,5 milles. Projet non économique en raison de l'insuffisance de revenus en regard de la mise de capital requise. " Dès 1946, SWP inclut cette ligne dans son programme de construction. Elle est partiellement construite en 1947, remise au programme de 1947-1948 et achevée en 1948-1949. AHQ, fonds de la CSPQ et Programmes d'électrification rurale de SWP.

74. "Client probable» est utilisé dans les rapports des compagnies et des commissions pour désigner les agriculteurs qui ont prévu de se raccorder à la ligne dès sa construction. Il n'est pas nécessairement égal aux clients effectivement raccordés à la fin de la construction. Le terme a une signification différente de "client possible", car, dans ce cas, on tient compte de tous les usagers qui pourraient être desservis par cette ligne; l'expression anglaise est « ultimate numbers of customers ». Selon nos calculs, AHQ, fonds P3, documents n ${ }^{\text {os }} 224,236$ et 237.

75. La situation est observable dans l'ensemble du Québec. La densité par mille de ligne diminue entre 1945 et 1960, passant de 7,9 à 6,5. Selon l'Office de l'électrification rurale, calculé par Y. Tremblay, op. cit., 514. 
néanmoins encore patienter. Les délais seront cependant moins importants qu'en périphérie du Québec ${ }^{76}$ (en Abitibi ou au Bas-Saint-Laurent, par exemple). Dès 1949-1951, les distributeurs raccorderont graduellement les dernières paroisses. Au milieu des années 1950, l'électrification des comtés de Nicolet et de Yamaska est pour ainsi dire complétée et, en 1961, les exploitations agricoles qui demeurent dépourvues du service sont alors des cas isolés. En 1961, en effet, 99,4\% des exploitations agricoles de Nicolet sont électrifiées alors que, dans Yamaska, 97\% d'entre elles le sont.

\section{CONCLUSION}

Plus de quarante années se seront écoulées entre l'apparition des premiers embryons de réseaux dans les campagnes québécoises et le moment où est réalisée l'électrification des fermes sur l'ensemble du territoire. D'abord jugée accessoire, voire bien de luxe, l'électricité acquiert progressivement pour les ruraux le statut de service public essentiel. C'est durant l'entre-deux-guerres que s'accomplit ce passage décisif. Mais les conditions sociales et économiques ne sont pas encore réunies pour que les distributeurs acceptent d'investir massivement dans la construction de lignes rurales, si bien que jusqu'en 1945 l'électrification des campagnes au Québec accuse un retard croissant par rapport à l'Ontario. Dans cet article, nous avons voulu montrer que l'électrification rurale obéit, jusqu'au milieu des années 1940, à un rapport de forces triangulaire: la population, qui la réclame comme un droit; les entreprises électriques, qui poursuivent des objectifs de rentabilité à court terme et négligent pour cela le milieu rural; et l'État, qui arrive mal à définir son propre rôle en la matière, qui tente de concilier les intérêts des uns et des autres et qui temporise.

Les réformes introduites pendant la guerre par le gouvernement Godbout annoncent le nouveau contexte qui prévaudra après 1945 et qui, malgré le retour au pouvoir d'un gouvernement peu interventionniste, sera néanmoins favorable à l'électrification massive des campagnes. Certes, l'ambitieux programme d'extension des réseaux ruraux,

76. À l'échelle du Québec, encore en 1961, plusieurs centaines d'exploitations agricoles situées en périphérie ne sont toujours pas électrifiées. En Gaspésie et aux Îles-de-la-Madeleine, encore $12,5 \%$ des fermes occupées n'ont pas l'électricité. Dans le comté de Hull-Gatineau, c'est plus de 200 exploitations agricoles qui sont dépourvues d'électricité. Dans Pontiac, l'indice d'électrification rurale ne dépasse pas $75 \%$ et dans Labelle, il est de $88 \%$. RDC, 1961. 
confié par l'État à Hydro-Québec lors de sa création, est abandonné au profit d'une approche fondée sur la complémentarité des secteurs privé, municipal et coopératif. Le découpage du territoire en zone d'intervention laisse grosso modo le Québec central aux grands distributeurs privés, et les régions plus périphériques aux coopératives d'électricité, soutenues par l'action d'un nouvel organisme gouvernemental, l'Office de l'électrification rurale. Le compromis duplessiste, ce fragile équilibre entre des forces sociales aux intérêts depuis longtemps divergents, créé néanmoins des conditions propices à la réalisation d'ambitieux programmes d'extension des lignes rurales au sortir de la guerre.

Mais ce sont bien autant les changements socio-économiques de l'après-guerre qui ont permis l'électrification accélérée du monde rural. L'utilisation accrue de l'électricité par les abonnés urbains et les autres catégories d'usagers, conjuguée à la forte croissance économique et à l'augmentation des revenus agricoles incitent les principales entreprises d'électricité à investir les capitaux nécessaires à l'électrification des campagnes. Si elles paraissent d'abord vouloir préserver leur monopole territorial des empiètements des coopératives, elles en viennent bientôt à envisager le milieu rural comme un véritable marché à développer et acceptent pour cela de revoir leur approche jusque-là fondée sur des critères de stricte rentabilité à court terme. Ces questions font l'objet de nos présentes recherches.

L'analyse régionale de la diffusion de l'électricité en milieu rural révèle ce changement d'attitude. Plusieurs comtés, peu électrifiés en 1931, sont presque entièrement desservis en 1951. Cette étude montre également que l'électrification rurale est un processus très différencié selon les régions. L'examen à l'échelle des paroisses des comtés de Nicolet et de Yamaska nous a permis d'en expliciter le processus. Avant 1945, la proximité des centres urbains et industriels - où sont concentrées les principales infrastructures de transport et de distribution d'énergie électrique - semble déterminante pour la diffusion de l'électricité à la campagne. Les distributeurs prolongent alors les réseaux urbains existants vers les marchés ruraux les plus prometteurs. Et ces lignes sont construites selon un programme qui tient peu compte des demandes des cultivateurs. Après 1945, en revanche, les ajouts au réseau sont prioritairement effectués là où des coopératives ont été fondées et dans les rangs où des cultivateurs réclament l'électricité depuis longtemps. À un modèle de diffusion qui procède par grandes branches à partir d'un tronc principal (le réseau de transport d'énergie interurbain) 
vers les secteurs les plus proches et les plus rentables s'en est donc substitué un autre plus ramifié, plus éclaté pourrait-on dire.

$\mathrm{Au}$ total, les compagnies privées construiront, à l'échelle du Québec, plus de lignes que leurs concurrentes coopératives. Ces dernières ont néanmoins joué un rôle crucial en stimulant, comme nous l'avons dit, l'électrification rurale par l'entreprise privée et en construisant ellesmêmes des milliers de milles de lignes. Les modes de fonctionnement des coopératives et leurs rapports avec les organismes gouvernementaux demeurent encore mal connus cependant. Nous nous employons à examiner cette question dans nos recherches actuelles. Il en va de même pour le problème des liens entre l'électricité et la modernisation de l'agriculture et celui de l'impact sur le mode de vie des ruraux, sujet presque complètement ignoré dans les travaux sur l'histoire de l'électricité parus jusqu'ici. Ces dernières remarques nous montrent tout l'intérêt qu'il y a à poursuivre cette enquête en prenant aussi en compte l'univers des représentations. 
ANNEXE

Les principales lignes de transport de la rive sud de Trois-Rivières, en 1929

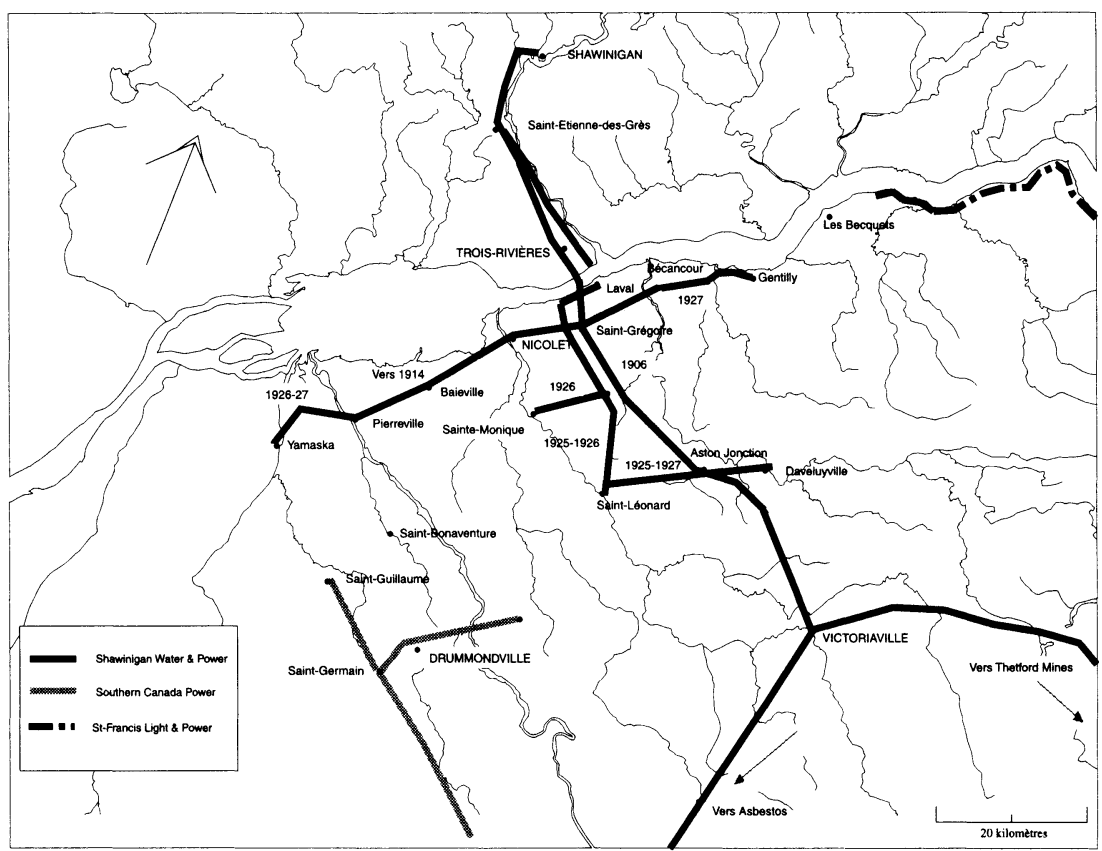

Sources : Central Electric Station in Canada, 1922 et 1928 et SWP, 25 années de progrès. 